\title{
Retinal Neurons Curb Inflammation and Enhance Revascularization in Ischemic Retinopathies via Proteinase-Activated Receptor-2
}

Nicholas Sitaras, ${ }^{* \dagger}$ José Carlos Rivera, ${ }^{* \dagger}$ Baraa Noueihed, ${ }^{\ddagger}$ Milsa Bien-Aimé, ${ }^{\dagger}$ Karine Zaniolo, ${ }^{\S}$ Samy Omri, ${ }^{\dagger \dagger}$ David Hamel, ${ }^{\star}$ Tang Zhu, ${ }^{*}$ Pierre Hardy, ${ }^{\llbracket}$ Przemyslaw Sapieha, ${ }^{\dagger}$ Jean-Sébastien Joyal, ${ }^{* \star \pi}$ and Sylvain Chemtob ${ }^{* \dagger \neq \uparrow}$

From the Departments of Pharmacology* and Pediatrics, ${ }^{\circledR}$ CHU Sainte-Justine Hospital, University of Montréal, Montréal; the Department of Ophthalmology, ${ }^{\dagger}$ Maisonneuve-Rosemont Hospital Research Center, University of Montréal, Montréal; the Department of Pharmacology and Therapeutics, ${ }^{\ddagger}$ McGill University, Montréal; and the LOEX-CUO Research Center, ${ }^{\S}$ Saint-Sacrement Hospital, Québec, Québec, Canada

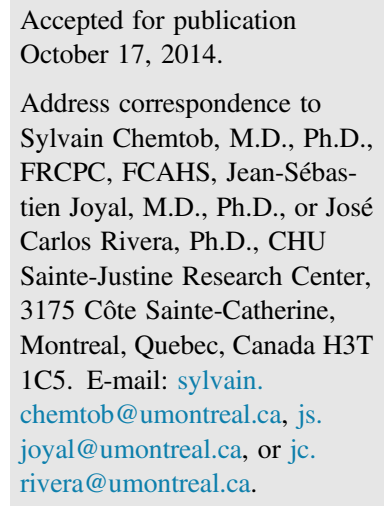

\begin{abstract}
Ischemic retinopathies are characterized by sequential vaso-obliteration followed by abnormal intravitreal neovascularization predisposing patients to retinal detachment and blindness. Ischemic retinopathies are associated with robust inflammation that leads to generation of IL-1 $\beta$, which causes vascular degeneration and impairs retinal revascularization in part through the liberation of repulsive guidance cue semaphorin 3A (Sema3A). However, retinal revascularization begins as inflammation culminates in ischemic retinopathies. Because inflammation leads to activation of proteases involved in the formation of vasculature, we hypothesized that proteinase-activated receptor (Par)-2 (official name F2rl1) may modulate deleterious effects of IL-1 $\beta$. Par2, detected mostly in retinal ganglion cells, was up-regulated in oxygen-induced retinopathy. Surprisingly, oxygen-induced retinopathy-induced vasoobliteration and neovascularization were unaltered in Par2 knockout mice, suggesting compensatory mechanisms. We therefore conditionally knocked down retinal Par2 with shRNA-Par2-encoded lentivirus. Par2 knockdown interfered with normal revascularization, resulting in pronounced intravitreal neovascularization; conversely, the Par2 agonist peptide (SLIGRL) accelerated normal revascularization. In vitro and in vivo exploration of mechanisms revealed that IL-1 $\beta$ induced Par2 expression, which in turn down-regulated sequentially IL-1 receptor type I and Sema3A expression through Erk/Jnkdependent processes. Collectively, our findings unveil an important mechanism by which IL- $1 \beta$ regulates its own endothelial cytotoxic actions by augmenting neuronal Par2 expression to repress sequentially IL-1 receptor type I and Sema3A expression. Timely activation of Par2 may be a promising therapeutic avenue in ischemic retinopathies. (Am J Pathol 2015, 185: 581-595; http://dx.doi.org/ 10.1016/j.ajpath.2014.10.020)
\end{abstract}

Ischemic retinopathies, such as retinopathy of prematurity and in some circumstances diabetic retinopathy, are the main causes of severe visual impairment in children and working class populations in industrialized countries. ${ }^{1,2}$
Ischemic retinopathies are biphasic diseases characterized by a degeneration of the retinal vascular bed, resulting in tissue hypoxia that triggers a compensatory albeit anarchic and deregulated vessel proliferation into the vitreous, which

\footnotetext{
Supported in part by Canadian Institutes of Health Research grant MOP12532 (S.C.). N.S., B.N., and S.O. are supported by studentship from the Fond de Recherche en Santé du Québec. D.H. is supported by a studentship from Centre Hospitalier Sainte-Justine. J.C.R. is supported by a fellowship from the Heart and Stroke Foundation of Canada and the Canadian Stroke Network. K.Z. is a recipient of a fellowship award from Foundation Fighting Blindness. P.S. holds a Canada Research Chair (Cell and Molecular Vision) and is supported by grants from Canadian Institutes
}

of Health Research. J.-S.J. is supported by a Burrough Wellcome Fund Career Awards for Medical Scientists and Foundation Fighting Blindness and holds a Career Development Award from the Canadian Child Health Clinician Scientist Program, a training initiative of the Canadian Institutes of Health Research. S.C. holds a Canada Research Chair (Vision Science) and the Leopoldine Wolfe Chair in translational research in age-related macular degeneration.

Disclosures: None declared. 
in severe instances can result in retinal detachment and blindness. ${ }^{3}$ Importantly, retinal ischemia from vascular decay also causes neuronal dysfunction and demise. ${ }^{4}$ Therefore, therapeutic interventions that help alleviate ischemic stress of the neural retina would be desirable to prevent both vascular and neuronal adverse consequences in patients with ischemic retinopathies.

In ischemic retinopathies, oxidative stress elicits microglia and resident macrophages to release inflammatory cytokines, ${ }^{5-8}$ notably IL-1 $\beta$, which further recruits and activates inflammatory cells thereby exacerbating retinal inflammation. ${ }^{8-10} \mathrm{We}$ found in a murine model of oxygeninduced retinopathy (OIR) that hyperoxia-activated microglia secretes IL-1 $\beta$ through an autocrine feedback loop. ${ }^{8}$ $\mathrm{IL}-1 \beta$, in turn, induces the release of repulsive cue semaphorin $3 \mathrm{~A}$ (Sema3A) from neighboring retinal ganglion cells (RGCs), which contributes to vaso-obliteration ${ }^{8}$ and hinders normal revascularization. ${ }^{11}$ This self-sustained and potentially unrelenting destructive inflammatory cascade, if kept unchecked, could result in severe obliteration of the microvascular network of the retina; however, this is not the case because revascularization eventually occurs, albeit slowly (during several days). ${ }^{12}$ Classic homologous down-regulation of IL receptor type I (Il-1RI) by its own natural ligand has not been observed; in fact, the contrary generally occurs. ${ }^{13,14}$ Thus, it is reasonable to postulate the existence of an alternate intrinsic regulatory mechanism that counteracts the self-sustained and potentially harmful IL-1 $\beta$-induced inflammatory cascade.

Inflammation is associated with the release and activation of various proteases involved in ensued vascularization. ${ }^{15}$ Proteinase-activated receptors (Pars) are G proteincoupled receptors activated by circulating proteases via cleavage of their $\mathrm{N}$-terminus, thus revealing a tethered ligand, which subsequently binds to their second extracellular loop to elicit intracellular signaling. ${ }^{16}$ Of the four members of the proteinase-activated receptor (Par) family (Par1 through Par4), ${ }^{16}$ Par2 (official name F2rl1) is recognized for its marked proangiogenic properties in the retina. ${ }^{17,18}$ Interestingly, Par2 is robustly activated by IL-1 $\beta$ in different cell types, including neurons and endothelial cells ${ }^{19-22}$; IL-1 $\beta$-mediated inflammation induces the expression of various proteases, which can cleave and activate Par2. ${ }^{23}$ This may appear rather counterintuitive because amplified IL- $1 \beta$ increases endothelial cell death in OIR, ${ }^{8,11}$ opposing the angiogenic effects of Par2. We therefore hypothesize that in OIR Par2 exerts its angiogenic properties by countering the cytotoxic actions of IL- $1 \beta$.

Using a mouse model of OIR, ${ }^{24}$ which mimics the cardinal features of ischemic retinopathies, we have uncovered a new mechanism of action for Par2 in retinal revascularization. By conditionally knocking down Par2 [using a lentiviral (Lv)encoded short hairpin (sh) RNA], we found that IL-1 $\beta$ regulates neuronal Par2 expression, which in turn reduces oxygen-induced vaso-obliteration and enhances desirable revascularization of the retina. We found that Par2 down-regulates IL-1RI specifically in RGCs, which in turn curtails the secretion of Sema3A, thereby facilitating retinal revascularization. Our findings unveil a novel property for Par2 in modulating inflammation, which in the context of ischemic retinopathies limits the vaso-obliterative effects of amplified $\mathrm{IL}-1 \beta$, allowing desirable revascularization and consequently reducing pathologic intravitreal neovascularization.

\section{Materials and Methods}

\section{Animals}

Adult C57BL6/J mice [wild type (WT)] and Par2 knockout mice (B6.Cg-F2rl1 $1^{\text {tm1 Mslb }}$ or Par2 ${ }^{-/-}$) were purchased from Jackson Laboratories (Bar Harbor, ME). Par2 ${ }^{-l-}$ mice were genotyped as described by Jackson Laboratories. Adoptive CD-1 lactating females were purchased from Charles Rivers Laboratories (Sainte-Hyacinthe, Canada) to tend to hyperoxiaexposed mice pups. All experiments were conducted in accordance with the Association for Research in Vision and Ophthalmology statement regarding use of animals in ophthalmic and vision research and were approved by Maisonneuve-Rosemont and Sainte-Justine Research Center animal care committees.

\section{OIR}

Mice pups were exposed from postnatal day 7 to postnatal day 12 to $75 \%$ oxygen using a BioSpherix oxycycler (BioSpherix, Lacona, NY). Vaso-obliteration and neovascularization were assessed in hyperoxia-exposed mice pups at postnatal days 12 and 17 , respectively, as described previously. ${ }^{24,25}$ Concisely, mice pups were fully anesthetized in 3\% isoflurane in oxygen and decapitated using a guillotine. Eyes were enucleated and fixed in $4 \%$ paraformaldehyde solution for 1 hour at room temperature. Retinas were dissected and stained overnight at $4^{\circ} \mathrm{C}$ with fluorescein-labeled Griffonia (Bandeiraea) simplicifolia lectin 1, isolectin B4 (Vector Labs, Burlingame, CA; 1:100). Lectin-stained retinas were whole mounted onto Superfrost/Plus microscope slides (Thermo Fisher Scientific, Waltham, MA) with the photoreceptor side down and imbedded in Fluoro-gel (Electron Microscopy Sciences, Hatfield, PA) and imaged at $10 \times$ using a Zeiss AxioObserver.Z1 (Zeiss, San Diego, CA). Images were merged into a single file using the MosiaX option in the AxioVision software version 4.6.5 (Zeiss). Quantification of vasoobliteration and neovascularization was assessed using the SWIFT_NV methods as previously described. ${ }^{26}$ The commercial IL-1 receptor antagonist (IL-1Ra) anakinra (Kineret; Swedish Orphan Biovitrum AB, Stockholm, Sweden) was administered intraperitoneally from postoperative day 7 (P7) to $\mathrm{P} 8$ at $20 \mathrm{mg} / \mathrm{kg} / \mathrm{d}$.

\section{Preparation of Lv Particles}

Third-generation Lv (HIV-1) was prepared as previously described. ${ }^{27}$ A p24 enzyme-linked immunosorbent assay kit 
(Clontech, Mountain View, CA) was used to quantify $\mathrm{Lv}$ titers for $\mathrm{Lv}$ shRNA against green fluorescent protein (GFP) $(8.5 \mathrm{ng} / \mu \mathrm{L}), \mathrm{Lv}$ shPar2 $(9.6 \mathrm{ng} / \mu \mathrm{L})$, and $\mathrm{Lv}$ GFP $(15.0 \mathrm{ng} / \mu \mathrm{L})$.

\section{Intravitreal Injections}

Animals were anesthetized in 3\% isolfurane in oxygen and injected intravitreally either at $\mathrm{P} 3$ with $1.0 \mu \mathrm{L}$ of $\mathrm{Lv}$ particles (described above) or at P7 with $10 \mu \mathrm{mol}$ of anakinra or P7 and P9 or P12 and P14 with $100 \mu \mathrm{mol}$ of $\mathrm{NH}_{2}$-SLIGRL (Elim Biopharm, Hayward, CA) using a Hamilton syringe equipped with 50-gauge glass capillary.

\section{Immunohistochemistry}

Eyes were enucleated from mice pups and fixed in 4\% paraformaldehyde at room temperature for 2 hours and saturated overnight at $4^{\circ} \mathrm{C}$ in a $30 \%$ sucrose solution before embedding in optimal cutting temperature compound (Tissue-Tek, Sakura, Torrance, CA). Coronal sections of $10 \mu \mathrm{m}$ were sectioned using a Cryostat CM3050S (Leica Microsystems, Concord, Canada). Sections were subsequently washed with phosphate-buffered saline, blocked, and permeabilized for 1 hour at room temperature and subsequently incubated with fluorescein-labeled Griffonia (Bandeiraea) simplicifolia lectin 1, isolectin B4 (Vector Labs; 1:100) for retinal vasculature. Antibodies to rabbit $\beta$ III-tubulin (ECM Biosciences, Versailles, KY; 1:1000), mouse $\beta$ III-tubulin (Sigma-Aldrich, St Louis, MO; 1:1500), mouse Par2 (SAM11, Invitrogen, Carlsbad, CA; 1:500), rat CD31 (BD Biosciences, Franklin Lakes, NJ; 1:100), rabbit IL-1RI (Santa Cruz Biotechnology, Santa Cruz, CA; 1:400), rabbit Sema3A (Abcam, Cambridge, MA, 1:500), or rabbit vascular endothelial growth factor (VEGF) (Santa Cruz Biotechnology; 1:200), whereas fluoresceinated secondary antibodies (goat anti-mouse IgG Alexa Fluor 488, 594, and/or 647 and goat anti-rabbit IgG Alexa Fluor 488, 594, and/or 647; Invitrogen) were used for localization studies according to the manufacturer's recommendations. Samples were visualized using $30 \times$ or $60 \times$ objectives with an IX81 confocal microscope (Olympus, Richmond Hill, Canada), and images were obtained with Fluoview software version 3.1 (Olympus). The specificity of Par2 SAM11 monoclonal antibody was confirmed on sagittal sections from $\mathrm{Par} 2^{-l-}$ mice, revealing absence of immunoreactivity.

\section{Laser-Capture Microdissection}

Eyes were enucleated and immediately embedded in optimal cutting temperature compound and snap frozen in liquid nitrogen and subsequently cut into $16-\mu \mathrm{m}$ coronal sections onto MembraneSlide 1.0 PEN nuclease free slides (Zeiss). To visualize vessels, sections were prepared as previously described. ${ }^{11}$ Retinal sections were laser microdissected with the Zeiss (Observer.Z1) Palm Microbeam laser microscope system. Isolated retinal RNA was transcribed into cDNA for quantitative real-time PCR analysis (see $R T-P C R$ and Quantitative Real-Time PCR).

\section{Western Blot}

Eyes were enucleated and retinas dissected and placed into commercial radioimmunoprecipitation assay buffer (Cell Signaling Technology, Danvers, MA) and homogenized using Precellys 24 homogenizer (Bertin Technologies, Montigny-le-Bretonneux, France). Samples were centrifuged, and $30 \mu \mathrm{g}$ of pooled retinal lysate from two different animals was loaded on an SDS-PAGE gel and subsequently electroblotted onto either polyvinylidene difluoride or nitrocellulose membrane (BioRad, Hercules, CA). After blocking, the membranes were blotted with mouse antibody to Par2 (1:400, SAM11, Invitrogen), mouse antibody to $\beta$-actin (1:1000, Santa Cruz Biotechnology), rabbit antibody to VEGF (1:200, Santa Cruz Biotechnology), rabbit antibody to IL-1RI (1:400, Santa Cruz Biotechnology), goat antibody to IL-1 $\beta$ (1:400, R\&D Systems, Minneapolis, MN), rabbit antibody to Sema3A (1:1000, Abcam), rabbit antibody to total (1:500, Cell Signaling) or phosphorylated Irak1 (1:500, Sigma-Aldrich), rabbit or mouse antibody to total and phosphorylated Erk1/2 (1:1000, Cell Signaling), rabbit antibody to total and phosphorylated p38 (1:1000, Cell Signaling), or rabbit or mouse antibody to total and phosphorylated Jnk (1:1000, Cell Signaling). After washing, membranes were incubated with 1:5000 horseradish peroxidase-conjugated anti-mouse or 1:2000 horseradish peroxidase anti-goat or anti-rabbit secondary antibodies (Millipore, Billerica, MA). Membranes were imaged with LAS-3000 imager, and bands were assessed using densitometry plugins in Multi Gauge software version 4.0 (FujiFilm, Tokyo, Japan). Specificity of SAM11 antibody against Par2 was tested in lysates from $\mathrm{Par}^{-1-}$ mice retina using WT mice retina as control; immunoblots using Par2 SAM11 antibody on cell lysates from various cell lines expressing Par2 or not have also been provided.

\section{RT-PCR and Quantitative Real-Time PCR}

Freshly dissected whole retina or laser-capture microdissected samples were processed using RiboZol RNA Extraction Reagent (AMRESCO, Solon, Ohio) as indicated in the manufacturer's instructions. Genomic DNA was removed using DNase I (Invitrogen). Approximately $1 \mu \mathrm{g}$ of total RNA was reverse transcribed into cDNA using iScript RT Supermix (BioRad) as indicated in the manufacturer's instructions. cDNA was analyzed by quantitative real-time PCR using iQ SYBR Green Supermix (BioRad) with primers targeting mouse Par2 (forward 5'-TGACCACGGTCTTTCTTCCG-3' and reverse $5^{\prime}$-TCAGGGGGAACCAGATGACA-3'), rat Par2 (forward 5'-TGGGAGGTATCACCCTTCTG-3' and reverse 5'-GGGGAACCAGATGACAGAGA-3'), mouse Sema3A (forward 5'-GCTCCTGCTCCGTAGCCTGC-3' and reverse $5^{\prime}$-TCGGCGTTGCTTTCGGTCCC-3'), mouse 
A
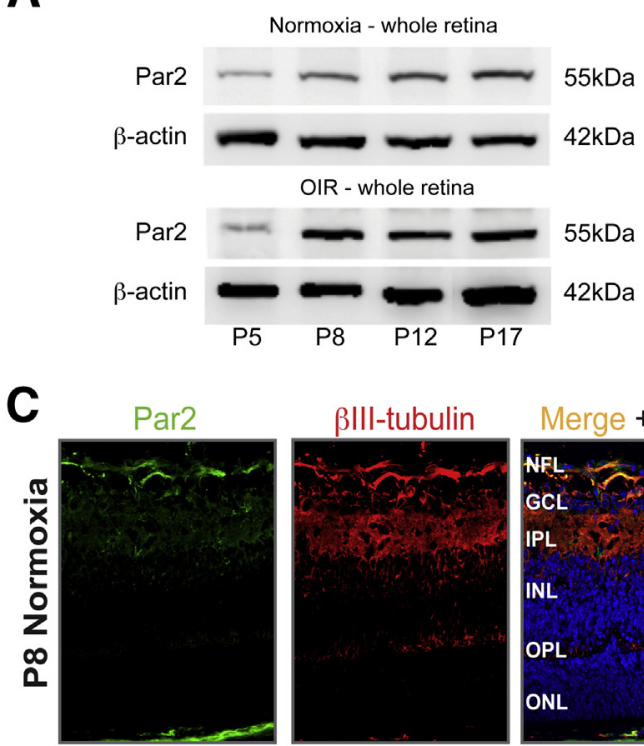

Par2

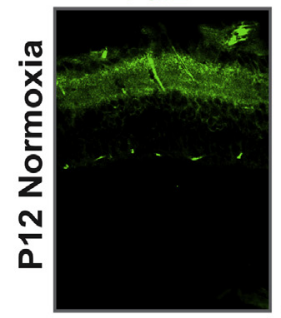

Par2

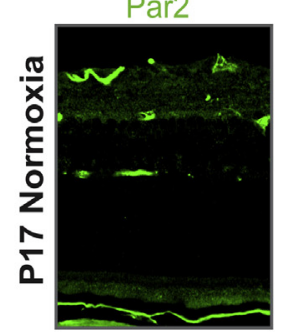

D

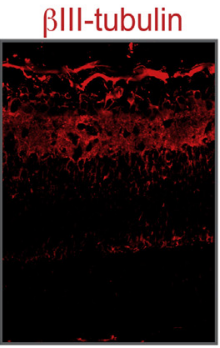

ßIII-tubulin

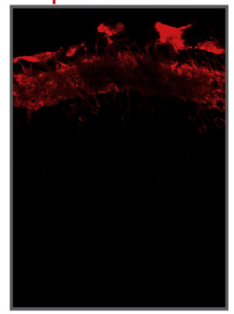

$\beta$ |ll-tubulin

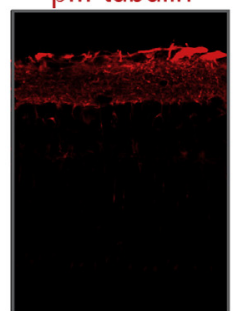

B
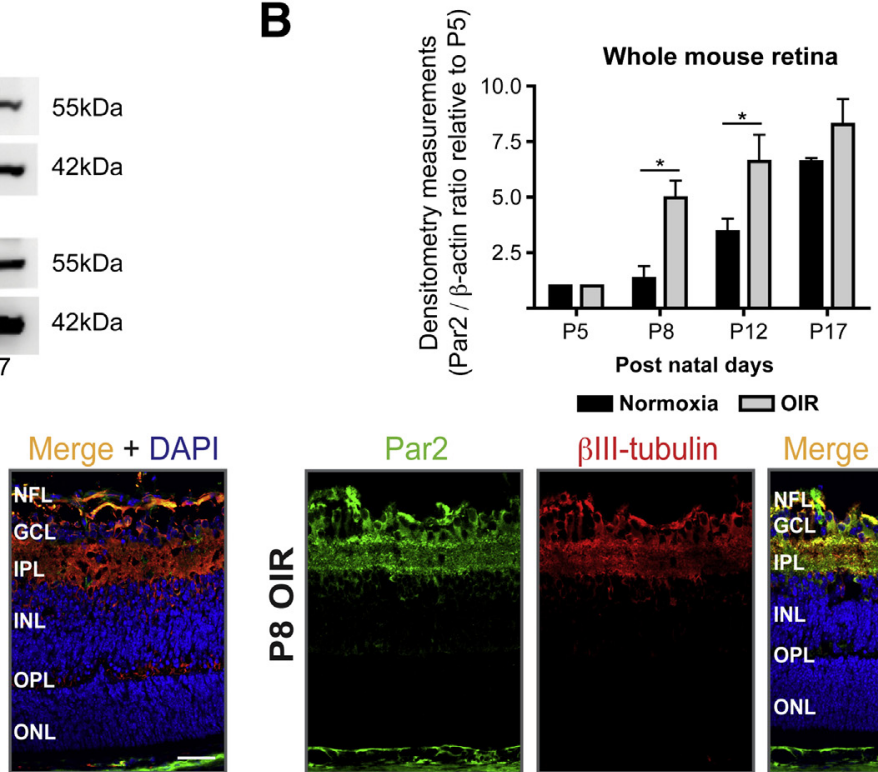

Merge + DAPI

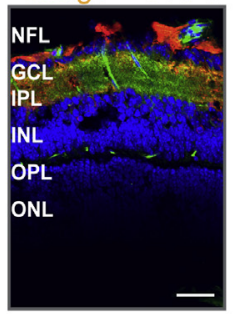

Merge + DAPI

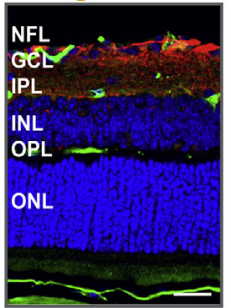

- Normoxia $\square$ OIR
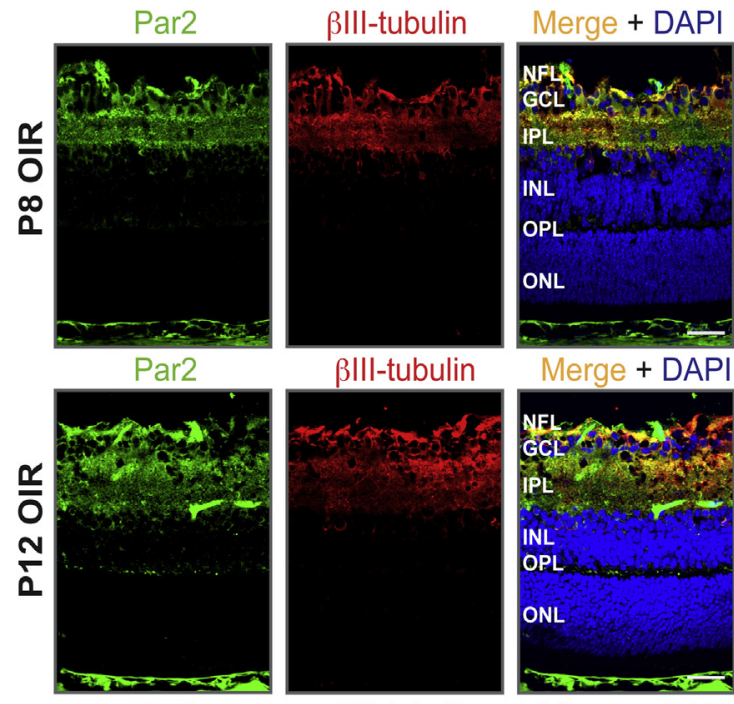

ßIII-tubulin

Merge + DAPI
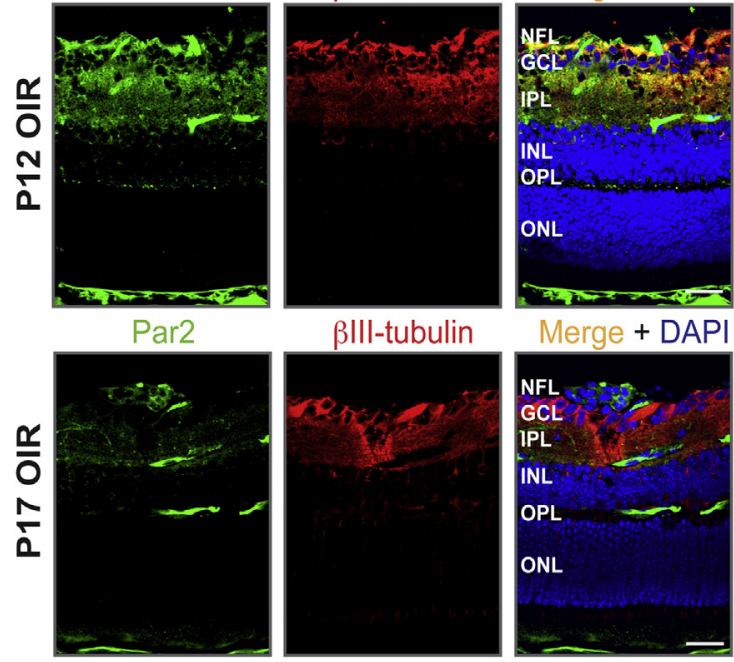

ßIII-tubulin
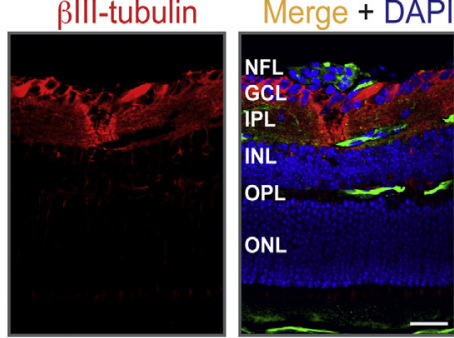

Par2 expression / cyclophilin A

(fold change relative to norm vessels)

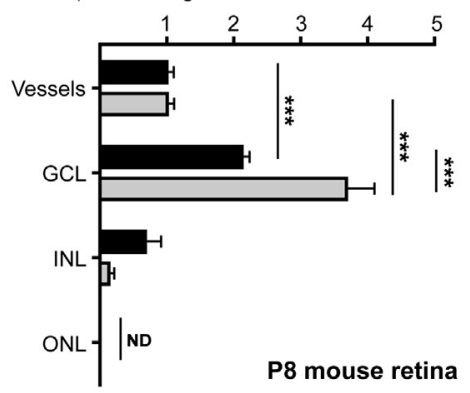

Figure 1 Neuronal proteinase-activated receptor (Par)-2 increases in mice retina during oxygen-induced retinopathy (OIR). A: Western blot analysis of wildtype mice whole retina at different time points of OIR showing increases of Par2 at postnatal day 8 (P8) and P12 compared with room-air raised mice retinas (two retinas per time point). B: Densitometry quantification of A. Values are normalized to P5 retinas. C: Immunohistochemical analysis on retinal coronal sections from P8, P12, and P17 wild-type mice pups exposed to either normoxia or OIR. Par2 (green) immunohistochemistry increases substantially in $\beta$ III-tubulin-expressing retinal ganglion cells (red) during 0IR at P8 and P12. Nuclei are counterstained with DAPI (blue). D: Laser-capture microdissected retinas from P8 pups exposed to normoxia or OIR. Quantitative real-time PCR on microdissected retinal layers reveal robust expression of Par2 mRNA in GCL, compared with microdissected vessels, which increases specifically in this layer after 24-hour exposure to high oxygen. ${ }^{*} P<0.05,{ }^{* *} P<0.001 . n=3$ independent experiments (A); $n=4$ (D). Scale bar $=50 \mu \mathrm{m}$ (C). Original magnification: $\times 300$ (C). GCL, ganglion cell layer; INL, inner nuclear layer; IPL, inner plexiform layer; $\mathrm{ND}$, not detected; $\mathrm{NFL}$, nerve fiber layer; $\mathrm{ONL}$, outer nuclear layer; $\mathrm{OPL}$, outer plexiform layer. 
VEGF-A (forward 5'-GCCCTGAGTCAAGAGGACAG-3' and reverse $5^{\prime}$-CTCCTAGGCCCCTCAGAAGT- ${ }^{\prime}$ ), mouse and rat IL-1RI (forward 5'-TGAATGTGGCTGAAGAGCAC$3^{\prime}$ and reverse $5^{\prime}$-CGTGACGTTGCAGACAGTT- $3^{\prime}$ ), and mouse IL- $1 \beta$ (forward 5'-CTGGTACATCAGCACCTCACA$3^{\prime}$ and reverse $5^{\prime}$-GAGCTCCTTAACATGCCCTG-3') [designed using Primer3 (National Center for Biotechnology Information)]. Quantitative gene expression analysis was assessed using the ABI 7500 Real-Time PCR system (Applied Biosystems, Foster City, CA) and compared with control genes cyclophilin A (forward 5'CAGACGCCACTGTCGCTTT- $3^{\prime}$ and reverse $5^{\prime}$-TGTCTTTGGAACTTTGTCTGCAA- $3^{\prime}$ ) or $18 \mathrm{~S}$ primers (Ambion, Austin, TX) using the $\Delta \Delta \mathrm{CT}$ quantification.

\section{Preparation of Stable Cell Lines}

The RGC-5 cell line was kindly provided by Neeraj Agarwal (National Eye Institute, Bethesda, MD), which was prepared as previously described. ${ }^{28}$ Undifferentiated RGC-5 samples were incubated overnight with Lv particles that contained shRNA. The next day, media was changed and incubated for 48 hours before selection with $5 \mu \mathrm{g} / \mathrm{mL}$ of puromycin (Sigma-Aldrich) for 7 days. Cells were differentiated thereafter with $1 \mu \mathrm{mol} / \mathrm{L}$ staurosporine for 1 hour (Sigma-Aldrich).

\section{Stimulation of RGC-5 Cell Lines and RBMVECs}

Rat brain microvascular endothelial cells (RBMVECs) were obtained from (Cell Applications Inc, San Diego, CA; catalog number R840-05a) and used between passages 2 and 7 (brain and retina microvascular endothelial cells share numerous common properties ${ }^{29}$ ). RGC-5 samples were cultured in Dulbecco's modified Eagle's medium (Invitrogen) supplemented with $10 \%$ fetal bovine serum (Cell Applications) and 1\% penicillin/streptomycin (Cell Applications) at $37^{\circ} \mathrm{C}$ and $5 \% \mathrm{CO}_{2}$ whereas RBMVECs were cultured as indicated in the manufacturer's instructions. Cells were starved 4 hours before treatment with 1.0 or $10.0 \mathrm{ng} / \mathrm{mL}$ of recombinant murine IL-1 $\beta$ (PeproTech, Rocky Hill, NJ) or 0,40 , or $100 \mu \mathrm{mol} / \mathrm{L}$ SLIGRL-NH 2 . Commercial inhibitors for Erk1/2 (U0126), Jnk (SP600125), and p38 (SB203580) were purchased from Sigma-Aldrich and used at $10 \mu \mathrm{mol} / \mathrm{L}$ approximately 60 minutes before SLIGRL- $\mathrm{NH}_{2}$ treatment.

\section{Preparation of RGC-5 Conditioned Media}

Terminally differentiated RGC-5 conditioned media were seeded $\left(10^{6}\right.$ cells $)$ and starved 4 hours before treatment with 0 or $100 \mu \mathrm{mol} / \mathrm{L} \mathrm{NH}_{2}$-SLIGRL and exposed to $5.0 \mathrm{ng} / \mathrm{mL}$ of recombinant murine IL-1 $\beta$ (PeproTech). Supernatants were collected 24 hours later, centrifuged briefly, and filtered through $0.22-\mu \mathrm{m}$ filters (Millipore) and distributed for proliferation assays (see below).

\section{Cell Survival Assay}

Approximately $10^{4}$ RBMVECs per well were seeded in 24-well plates and starved 4 hours before exposure to RGC-5 conditioned media. Neutralizing antibody to Sema3A was used at $2 \mu \mathrm{g} / \mathrm{mL}$ (Abcam). After 24 hours, 50 $\mu \mathrm{L}$ of a $5 \mu \mathrm{g} / \mathrm{mL}$ solution of thiazolyl blue tetrazolium bromide (Sigma-Aldrich) was added and cells incubated for 2 to 3 hours. Supernatants were aspirated and cells were lyzed and resuspended in acidified isopropanol. Duplicate absorbance readings were taken at $565 \mathrm{~nm}$ using an Infinite M1000 Pro plate reader (Tecan, San Jose, CA).

\section{Aortic Explant Microvascular Growth Assay}

Aortae were isolated from adult $\mathrm{Par} 2^{-1-}$ mice, sectioned into 1-mm rings, and placed into growth factor-reduced Matrigel (BD Biosciences) in 24-well plates. Rings were cultured in RBMVEC supplemented endothelial basal medium (Cell Applications Inc, San Diego, CA) 4 to 5 days before a 48 hours exposure to RGC-5 conditioned media. Treated rings were photomicrographed using AxioObserver (Zeiss) and microvascular growth assessed using Image Pro version 4.5 (Media Cybernetics, Rockville, MD). Neutralizing antibody to mouse $\mathrm{VEGF}_{164}$ was used at concentrations of $20 \mu \mathrm{g} / \mathrm{mL}$ (R\&D Systems).

\section{Statistical Analysis}

Results are presented as means \pm SEM. for all studies. Oneway or two-way analysis of variance with significance $\alpha=0.05$ was used for processing data. Bonferroni post hoc analysis was used for calculating significance between groups. Two-tailed Student's $t$-tests were used to test for significance between two means.

\section{Results}

\section{Neuronal Par2 Expression Augments during OIR}

Retinas from WT mice subjected to $75 \%$ oxygen for 5 days $(\mathrm{P} 7 \text { to } \mathrm{P} 12)^{24}$ were analyzed at different time points during OIR. During the early phases of vaso-obliteration (at P8), Par2 protein levels surged approximately threefold compared with normoxic controls (normalized versus P5) (Figure 1, A and B). Par2 expression in retina remained high during the hyperoxic phase at P12 (Figure 1, A and B); by P17, Par2 levels were comparable to normoxic controls. $\mathrm{Par}^{-1-}$ mice had no immunoreactivity to SAM11 Par2 monoclonal antibody (Supplemental Figure S1).

Immunofluorescence analysis on sagittal sections from P8 and P12 normoxic retinas had preferential distribution of Par2 in BIII-tubulin positive RGCs (Figure 1C and Supplemental Figure S2A), which robustly increased in these cells during OIR (Figure 1C and Supplemental Figure S2A). Par2 also slightly co-localized with retinal endothelial cells at P8 

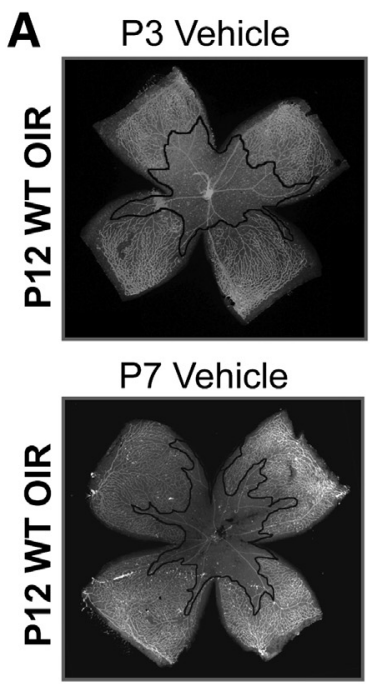

B
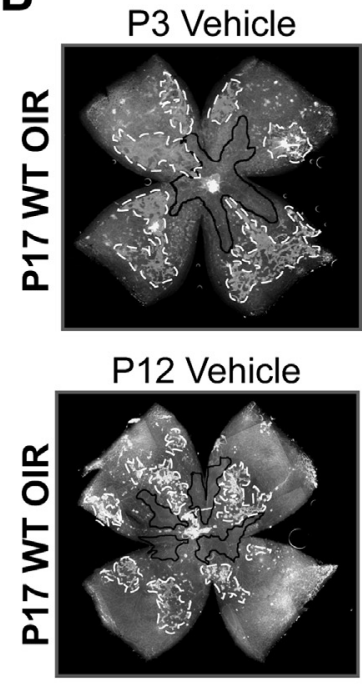

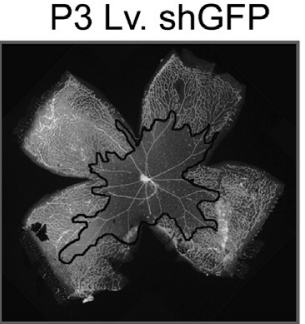

P7 SLIGRL

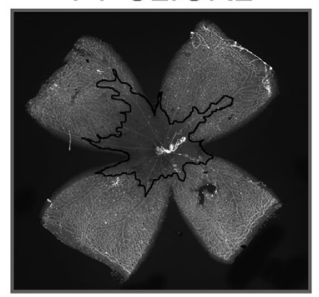

P3 Lv. shGFP

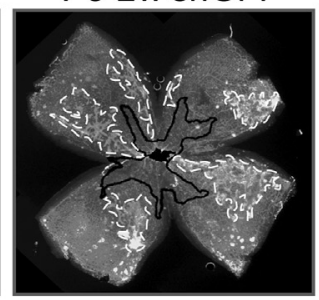

P12 SLIGRL

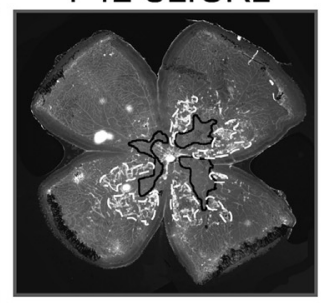

P3 Lv. shPar2

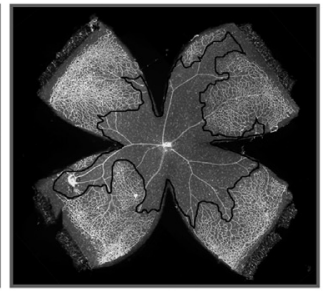

P7,9 SLIGRL

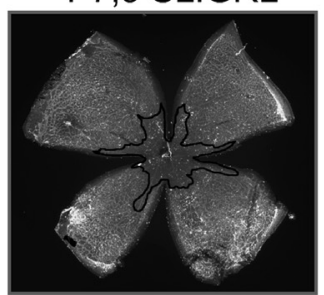

P3 Lv. shPar2

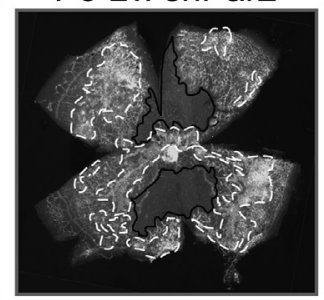

P12,14 SLIGRL

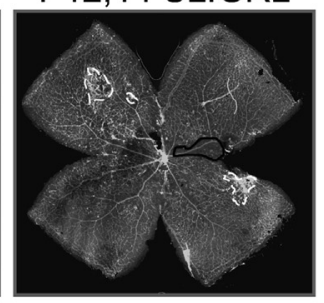

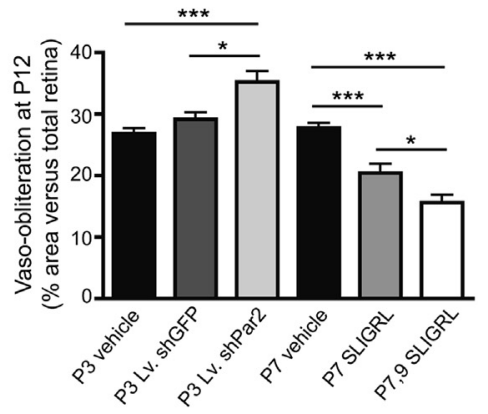
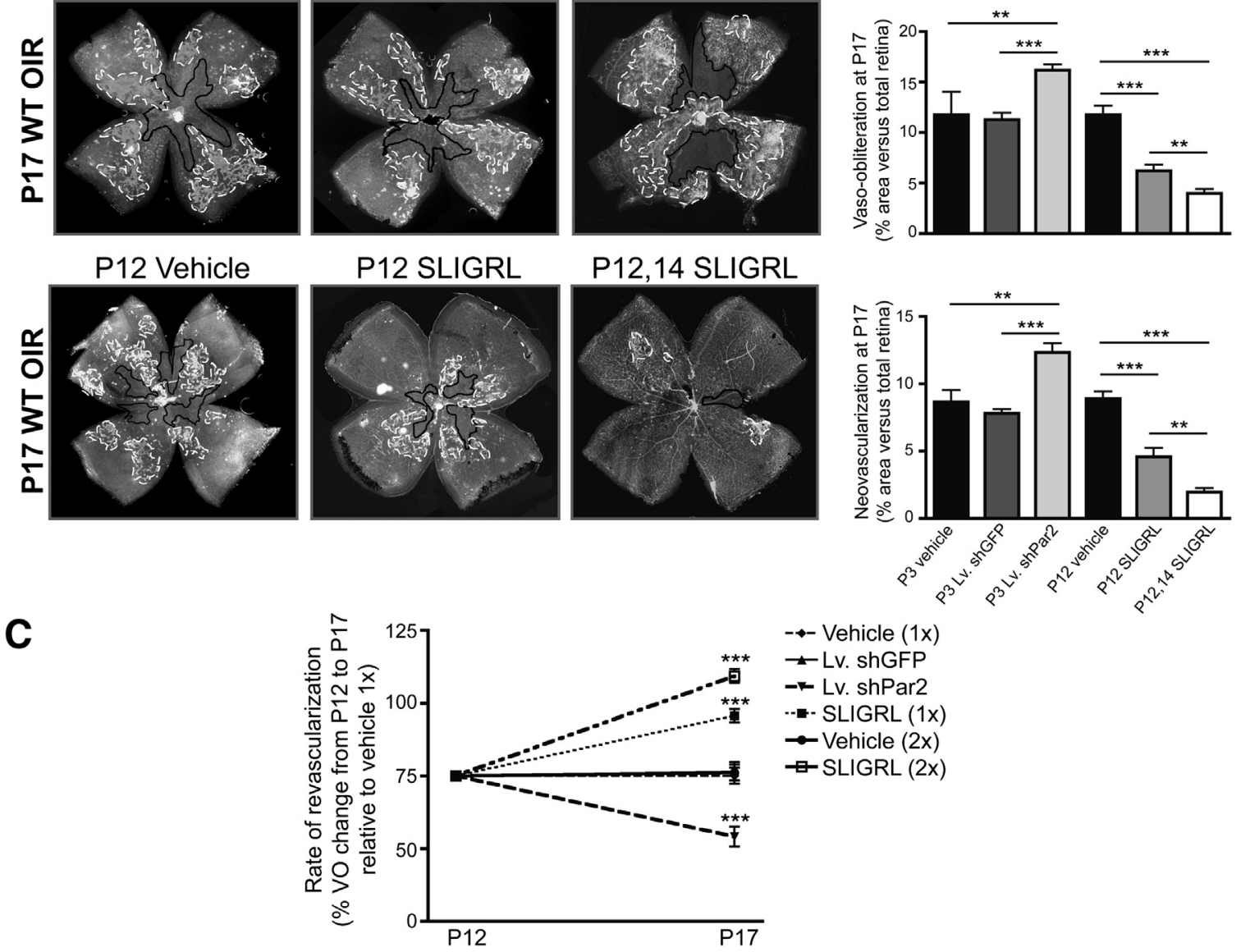

Figure 2 Modulation of proteinase-activated receptor (Par)-2 activity during oxygen-induced retinopathy (OIR) affects vaso-obliteration (V0) and neovascularization (NV). A: Representative photomicrographs from isolectin B4-stained retinal flat mounts from postnatal day 12 (P12) wild-type (WT) mice pups exposed to OIR. V0 areas are outlined with solid black lines. Pups injected intravitreally with LV containing shRNA against Par2 (approximately $9.6 \mathrm{ng}$ ) had significant increases in V0 compared with vehicle or control Lv shRNA against green fluorescent protein (shGFP) (approximately $8.5 \mathrm{ng}$ ) injected animals. Mice pups injected intravitreally with $100 \mu \mathrm{mol}$ Par2 agonist peptide SLIGRL-NH 2 , however, have a significant decrease in V0. V0 areas are represented in the bar graph on the right. B: Photomicrographs of P17 WT mice retina exposed to OIR and injected intravitreally with Lv shPar2 have increased V0 and NV compared with control vehicle or Lv shGFP-treated animals, whereas SLIGRL-treated mice pups have significant decreases in the avascular area and NV. Avascular areas and NV areas are outlined with solid black lines and white hashed lines, respectively. The bar graphs show the quantification of avascular areas and NV areas by SWIFT_NV. C: Retinal revascularization rate from P12 to P17 after OIR in mice previously injected with Lv shPar2 or SLIGRL. Change in V0 was calculated for each group using V0 at P12 as starting value (100\%). Samples at P17 were compared relative to singly injected vehicle-treated animals. Lv shPar2 delays whereas SLIGRL accelerates retinal revascularization. ${ }^{*} P<0.05,{ }^{* * P} P 0.01$, and ${ }^{* * *} P<0.001 . n=6$ to 12 retinas (A); $n=6$ to 12 retinas (B); $n=18$ to 23 retinas (C). Original magnification: $\times 100$ (A and B). 

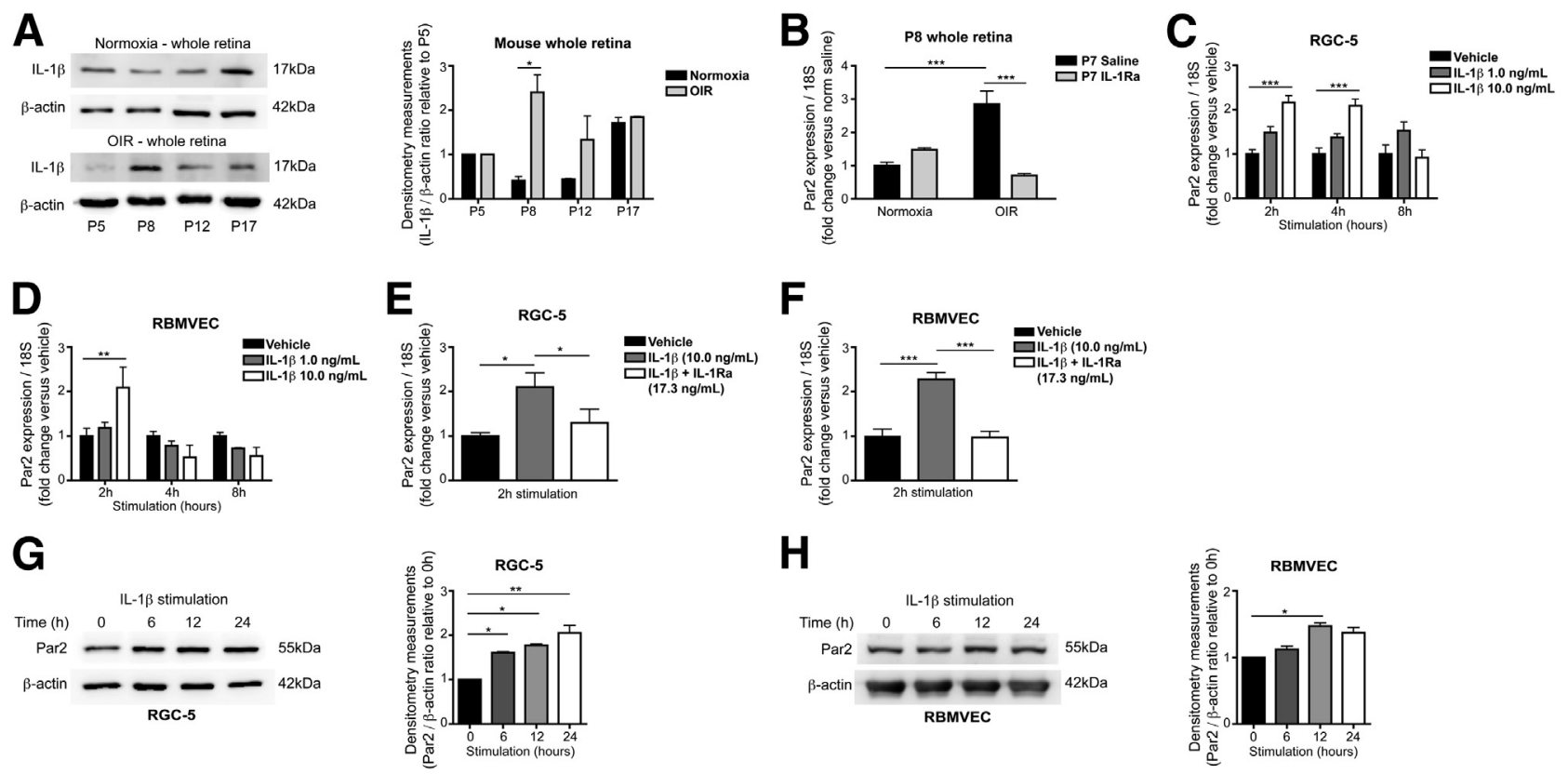

Figure 3 Increased expression of IL-1 $\beta$ in the retina during oxygen-induced retinopathy (OIR) stimulates proteinase-activated receptor (Par)-2 expression. A: Western blot analysis of IL-1 $\beta$ in whole retina from mice pups at various time points of OIR indicating an increase at postnatal day 8 (P8) compared with room-air raised wild-type (WT) mice pups (two retinas per time point). The bar graph shows the densitometry quantification. Values are normalized to P5 retinas. B: Quantitative real-time PCR analysis of whole retina from P8 WT mice pups demonstrating an increase in Par2 mRNA upon exposure to hyperoxia for 24 hours. Animals treated i.p. with IL-1 receptor antagonist (IL-1Ra; Kineret) have significantly less Par2 mRNA. IL-1 $\beta$ stimulated cultured retinal neurons [retinal ganglion cell (RGC)-5] (C) and rat brain microvascular endothelial cells (RBMVECs) (D) exhibit time- and dose-dependent increases in Par2 mRNA as analyzed by quantitative real-time PCR. Administration of IL-1Ra abrogates IL-1 $\beta$-dependent increases in Par2 mRNA in both RGC-5 (E) and RBMVECs (F) as determined by quantitative real-time PCR analysis. G: RGC- 5 samples stimulated with IL-1 $\beta$ have a time-dependent increase in Par2 protein levels. Densitometry quantifications are illustrated in the bar graphs. H: RBMVECs stimulated with IL-1 $\beta$ have an increase in Par2 protein levels at 12 hours only. The bar graph represent densitometry quantifications. $n=3$ independent experiments (A); $n=3(\mathbf{B}) ; n=4$ to 8 (C and $\mathbf{D}) ; n=2$ independent experiments $(\mathbf{G})$; $n=2$ independent experiments $(\mathbf{H})$. ${ }^{*} P<0.05,{ }^{*} P<0.01$, and ${ }^{*} * P<0.001$.

$\left(\mathrm{CD} 31^{+}\right)$(Supplemental Figure S2B). Laser-capture microdissection of retinal tissue followed by real-time quantitative PCR analysis revealed significantly higher Par2 mRNA in the ganglion cell layer (GCL) relative to microdissected vessels (Figure 1D and Supplemental Figure S2, C and D), particularly in OIR-subjected animals (approximately 3.5fold increase, $P<0.001$ ). Low levels of Par2 mRNA were also detected in the inner nuclear layer likely owing to the invading blood vessels that protrude into this layer in retinas from P8 mice pups (Figure 1D and Supplemental Figure S2, C and D); in OIR, the vaso-obliteration led to the disappearance of Par2 expression in this layer. At P17, Par2 expression was localized mostly to the retinal endothelium in both room air and hyperoxia-raised mice. Altogether, our data indicate that Par2 increases sharply during the early phases of OIR, and its expression is localized preferentially on RGCs.

\section{Par2 Protects the Retina from 0xygen-Induced Vaso-Obliteration and Induces Retinal Revascularization}

To explore the role of Par2 in ischemic retinopathy, we exposed Par2 knockout mice (Par2 ${ }^{-l-}$ ) to the OIR model. As previously reported, ${ }^{17}$ mice lacking the Par2 gene surprisingly had no change in vaso-obliteration or preretinal neovascularization compared with age-matched WT mice (Supplemental Figure S3A). Because, compensatory mechanisms are regularly reported with germ cell line gene modulation, ${ }^{30}$ and signaling redundancies exist among different Pars $^{16}$ (as reported in Par2 transgenic mice ${ }^{31}$ ), we attempted to circumvent this drawback by conditional knockdown of Par2 using Lv constructs bearing shRNAs targeting Par2 injected intravitreally in WT mice pups at P3. Lv vectors had high tropism for RGCs [see co-localization of green fluorescent protein (encoded in Lv) with BIII-tubulin] (Supplemental Figure S3B). ${ }^{11,28,32,33}$ In WT mice Lv shPar2 successfully down-regulated retinal Par2 expression by P7 compared with contralateral eyes injected with control Lv shGFP (Supplemental Figure S3C). Par2 knockdown caused an increase in retinal vaso-obliteration at P12 and P17 (Figure 2, A and B), resulting in a significant reduction in the rate of revascularization between these corresponding ages (by 20.9\%, $P<0.001$ ) (Figure 2C). Consequently, Lv shPar2-treated animals exhibited increased aberrant preretinal neovascularization at P17 compared with control Lv shGFP injected pups (Figure 2B); Lv shPar2 was ineffective in Par2 ${ }^{-/-}$pups (Supplemental Figure S4), consistent with the specificity of the shPar2. 

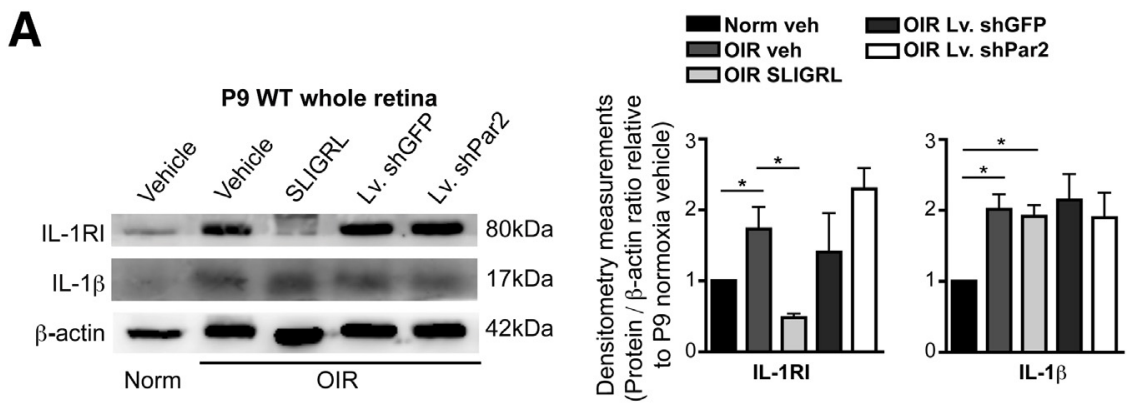

B

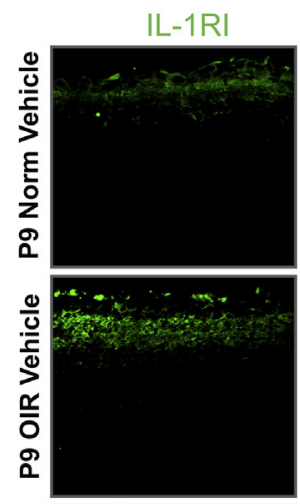

ßIII-tubulin

Merge + DAPI +
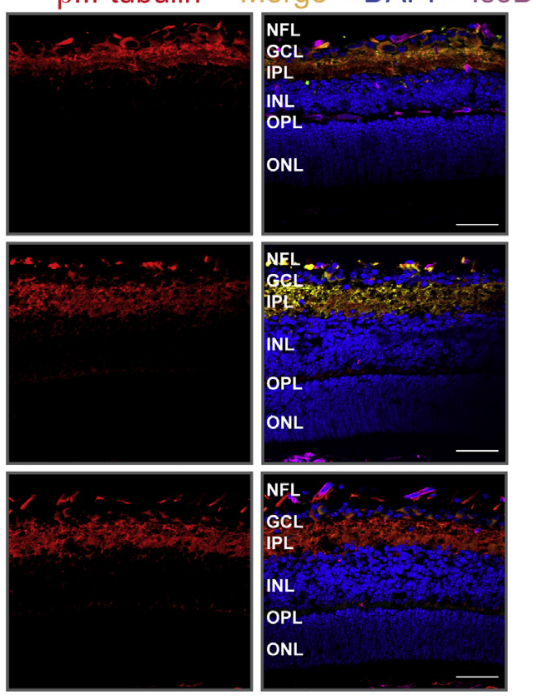

C
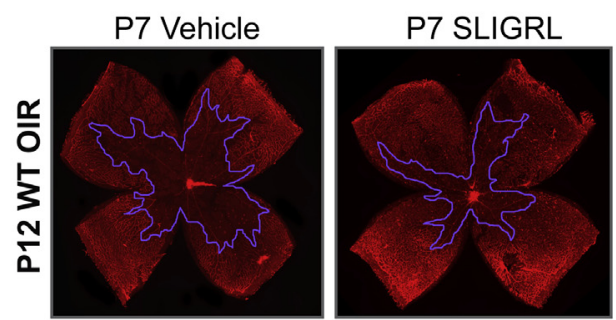

P7 IL-1Ra
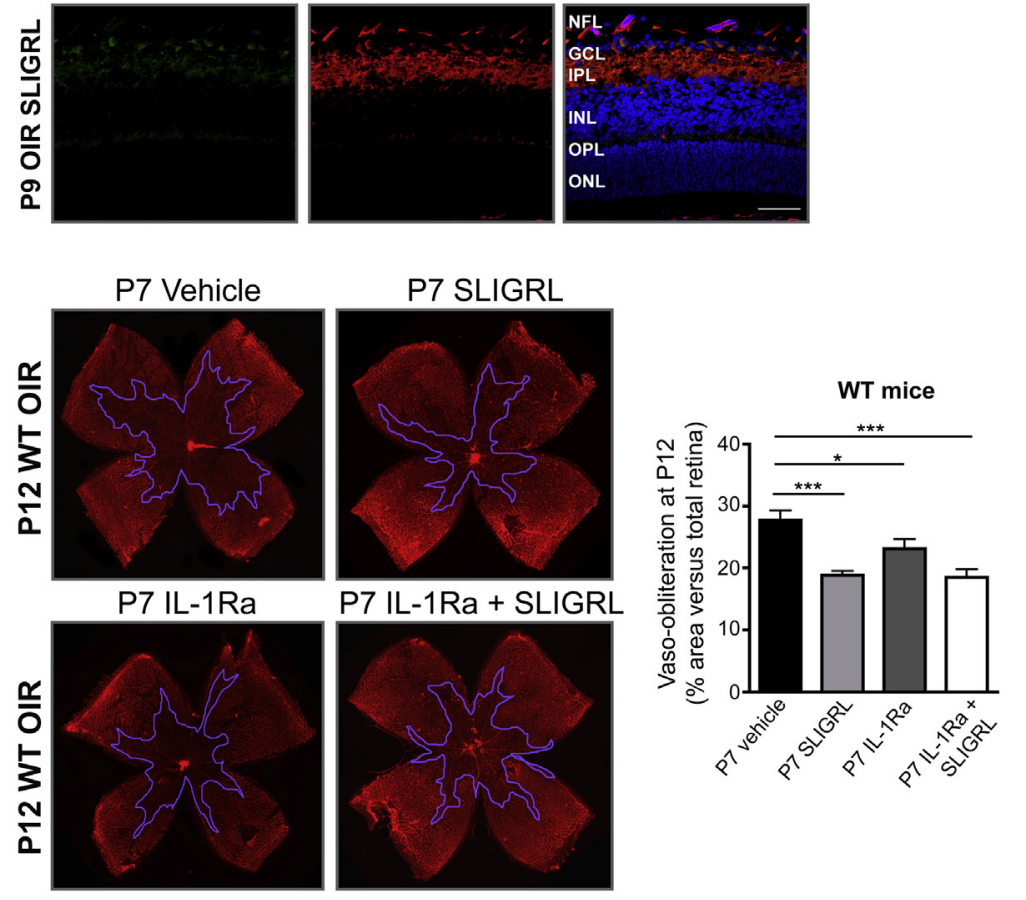

We next proceeded to evaluate the effects of exogenous Par2 stimulation with the Par2 activating peptide SLIGRL (corresponding to tethered ligand in mice) ${ }^{34}$ injected intravitreally. Because expression of Par2 increased robustly at $\mathrm{P} 8$ and $\mathrm{P} 12$, we administered SLIGRL via intravitreal injection during early (P7) and late (P12) hyperoxic exposure. Administration of SLIGRL at P7 caused a significant reduction in vaso-obliteration at P12 compared with vehicle-injected animals (Figure 2A); a successive injection at P9 further diminished vaso-

Figure 4 Proteinase-activated receptor (Par)-2 activation results in down-regulation of IL-1 receptor type I (IL-1RI) in vivo. A: Western Blot analysis of IL-1 $\beta$ and IL-1RI in postnatal day 9 (P9) wild-type (WT) mice whole retina exposed to oxygen-induced retinopathy (OIR) and injected with vehicle, 100 $\mu$ mol SLIGRL, Lv shRNA against green fluorescent protein (shGFP), or Lv ShPAR-2 (two retinas per group). Increases in IL-1 $\beta$ and IL-1RI are observed in OIR exposed retinas; however, treatment with SLIGRL significantly reduces IL-1RI expression but not IL- $1 \beta$. Conversely, administration of Lv shPar2 trends toward an increase in IL-1RI levels; however, the results are not significant. IL-1 $\beta$ levels remained the same. The bar graphs indicate densitometry quantification. B: Immunohistochemical analysis of P9 WT mice retinas exposed to OIR (middle panels) have significant immunoreactivity to IL-1RI (green) localized primarily in the ganglion cell layer ( $\beta$ III-tubulin; red) compared with normoxic-exposed retinas (upper panels). SLIGRL administration reverses this process (lower panels). Nuclei are counterstained with DAPI (blue) and vessels with isolection B4 (magenta). C: Representative images from isolectin B4-stained retinal flat mounts from P12 WT mice pups exposed to OIR and injected intravitreally with either $100 \mu \mathrm{mol}$ SLIGRL or $10 \mu \mathrm{mol}$ IL-1Ra or both. Vaso-obliteration (V0) areas are outlined with solid blue lines. Individually, administration of Par2 agonist and Il-1RI antagonist successfully inhibits hyperoxia-induced v0. However, there is no additive effect from coadministration of both SLIGRL and IL-1Ra. $n=3$ independent experiments (A); $n=6$ to 8 retinas (C). ${ }^{*} P<0.05,{ }^{* * *} P<0.001$. Scale bar $=50 \mu \mathrm{m}($ B). Original magnification: $\times 300(B) ; \times 100(C) . G C L$, ganglion cell layer; INL, inner nuclear layer; IPL, inner plexiform layer; $\mathrm{NFL}$, nerve fiber layer; $\mathrm{ONL}$, outer nuclear layer; $\mathrm{OPL}$, outer plexiform layer. obliteration (Figure 2A). Likewise, intravitreal injections of SLIGRL after hyperoxic exposure (P7-P12) at P12 and $\mathrm{P} 14$ reduced dose dependently the size of the avascular area measured at P17 (Figure 2B), which translated to an accelerated rate of revascularization between P12 and P17 (20.6\% and $34.3 \%$ for single and double injections, $P<0.001$ ) (Figure 2C). As expected, SLIGRL treatment diminished pathologic preretinal neovascularization at P17 (Figure 2B). Par2 ${ }^{-1-}$ mice were unresponsive to SLIGRL (Supplemental Figure S4). Collectively, these data indicate that Par2 


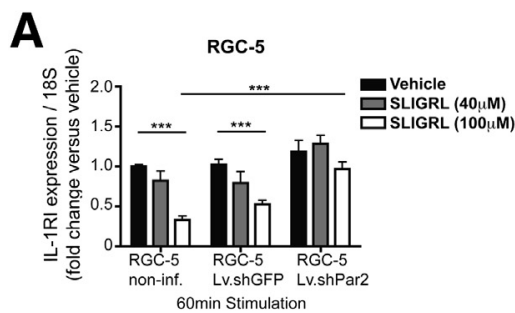

C

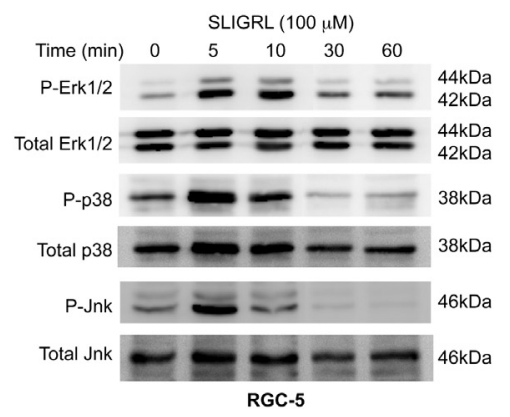

B

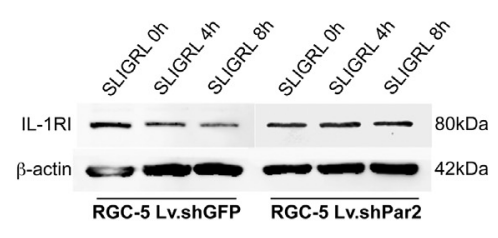

D

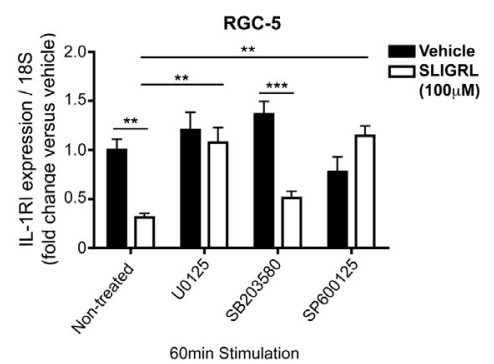

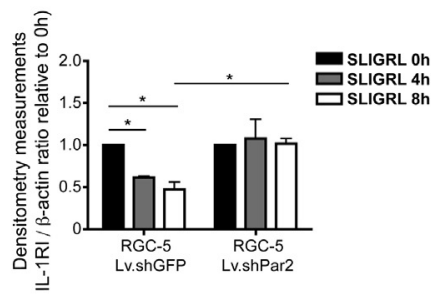

$\mathbf{E}$

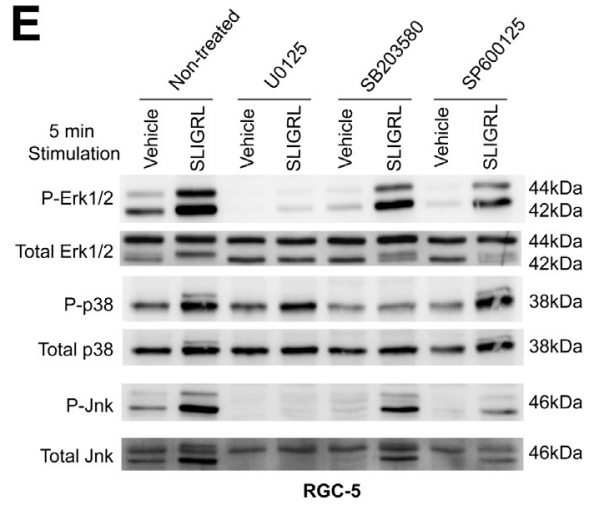

Figure 5 Erk1/2 activates downstream Jnk, which is required for proteinase-activated receptor (Par)-2-dependent inhibition IL-1 receptor type I (IL-1RI) in retinal neurons. A: Retinal ganglion cell (RGC)-5 cell lines exhibit dose-dependent decreases in IL-1RI mRNA after 1-hour stimulation with SLIGRL. RGC-5 infected with Lv shPar2 abrogates effect. Lv shGFP-infected cells served as control RGC-5. B: RGC-5 cell lines have a time-dependent decrease in IL-1RI protein levels after $100 \mu \mathrm{mol} / \mathrm{L}$ SLIGRL treatment; Lv shPar2-infected RGC-5 do not respond to SLIGRL treatment. Densitometry quantification is shown below. C: Administration of SLIGRL activates mitogen-activated protein kinase (MAPK) signaling pathways: Erk1/2, Jnk, and p38. D: RGC-5 cell lines pretreated with either MEK1/2 inhibitor (U0125) or Jnk inhibitor (SP600125) but not 338 inhibitor (SB203580) abrogate the SLIGRL-dependent decreases in IL-1RI mRNA in RGC-5. E: Activation of MAPK by $100 \mu \mathrm{mol} / \mathrm{L} \mathrm{SLIGRL} \mathrm{in} \mathrm{RGC-5} \mathrm{is} \mathrm{abolished} \mathrm{using} \mathrm{specific} \mathrm{inhibitors} \mathrm{to} \mathrm{MEK1/2} \mathrm{(U0125),} \mathrm{p38} \mathrm{(SB203580),} \mathrm{and} \mathrm{Jnk} \mathrm{(SP600125);} \mathrm{inhibition} \mathrm{of} \mathrm{Erk1/2} \mathrm{also} \mathrm{inhibits}$ Jnk phosphorylation. Conversely, Erk1/2 phosphorylation is not affected by Jnk inhibitor, indicating that Erk1/2 activates Jnk, which sequentially downregulates IL-1RI mRNA. $n=6$ to $10(\mathbf{A}) ; n=2$ independent experiments $(\mathbf{B})$. ${ }^{*} P<0.05,{ }^{*} * P<0.01$, and ${ }^{*} * *<0.001$.

enhances the integrity of the inflammation-challenged ${ }^{8,11}$ retinal vascular bed during hyperoxic stress by promoting normal revascularization, which in turn reduces undesirable aberrant preretinal neovascularization.

\section{IL-1 $\beta$ Induces Par2 Expression in Neuronal and Endothelial Cells}

Augmented levels of inflammatory cytokines, such as IL-1 $\beta$, IL-6, and tumor necrosis factor- $\alpha$, are found in the vitreous of patients with ischemic retinopathies and corresponding animal models. ${ }^{5-8,11}$ Of relevance, IL-1 $\beta$ increases Par2 expression in cultured endothelial cells, ${ }^{20}$ chondrocytes, ${ }^{21}$ synovial cells, ${ }^{19}$ and neuronal cells. ${ }^{22}$ We explored the association between IL-1 $\beta$ and Par2 in OIR. Consistent with the intimate link between oxidative stress and inflammation, ${ }^{35}$ IL-1 $\beta$ levels increased during hyperoxia, particularly at P8 (Figure 3A), when Par2 expression peaked in OIR (Figure 1). Pretreatment of hyperoxia-exposed mice with the IL-1 receptor antagonist (IL-1Ra) anakinra reduced $\mathrm{IL}-1 \beta$ mRNA expression (Supplemental Figure S5A) as reported $^{8}$ and significantly lowered Par2 mRNA levels at P8 (Figure 3B), suggesting that IL-1 $\beta$ may regulate Par2 expression in vivo.

In vivo findings were ascertained in vitro. Par2-expressing cultured murine retinal neuronal cells (RGC-5) and rat brain microvascular endothelial cells (RBMVECs) (Supplemental
Figure S5, B and C) had dose-dependent increases in Par2 mRNA expression on treatment with recombinant murine IL-1 $\beta$ (Figure 3, C and D); these effects were abolished by co-treatment with IL-1Ra (Figure 3, E and F). Par2 mRNA increased within 2 to 4 hours after stimulation with IL-1 $\beta$ in both RGC-5 and RBMVECs but was not sustained thereafter, whereas increased Par2 protein translation was detected by 6 to 12 hours in both cells but continued to increase at 24 hours only in RGC-5 (Figure 3, G and H), consistent with changes in Par2 expression in microdissected retinas of animals subjected or not to OIR (Figure 1D). Hence, IL-1 $\beta$ up-regulates Par2 expression in retinal ganglion neurons in a sustained manner but yields a limited and transient response in vascular endothelial cells.

\section{Par2 Is Involved in Negative Feedback Regulation of IL-1 Response}

Chronic inflammation can be detrimental to the nascent vessels, either directly via cytokine or chemokine signaling ${ }^{36-40}$ or indirectly through inflammatory cell activation. ${ }^{9,10,41} \mathrm{We}$ previously uncovered that IL-1 $\beta$, predominantly generated by microglia in OIR, interfered with normal retinal revascularization by eliciting RGCs to secrete Sema3A, a proapoptotic endothelial repulsive cue. ${ }^{8,11}$ Paradoxically, we found that IL-1 $\beta$ also up-regulates Par2 (Figure 3), which enhances 
A

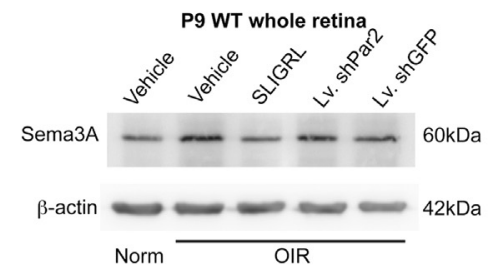
Norm veh
GOIR veh LoIR. shPar2 घOIR veh

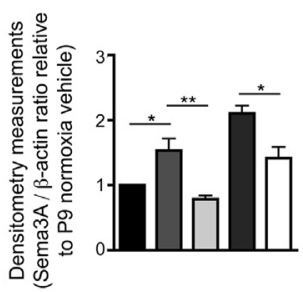

B

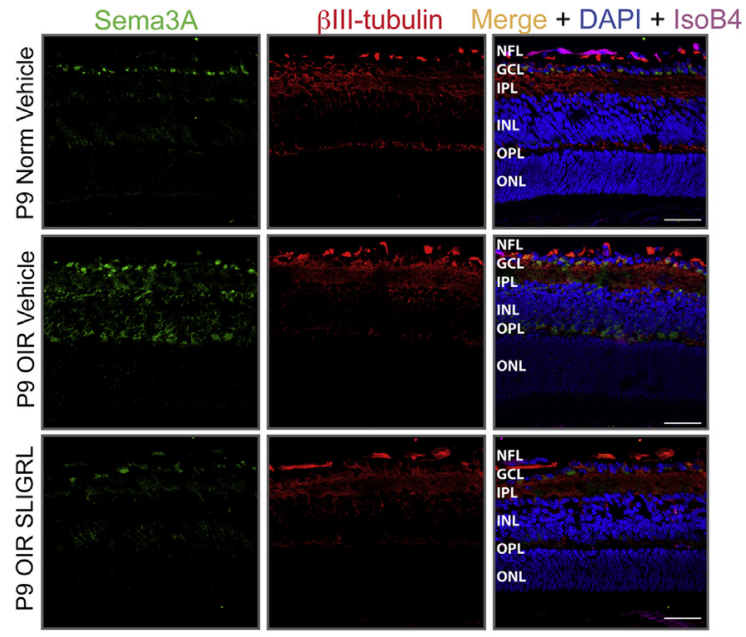

$\mathbf{E}$

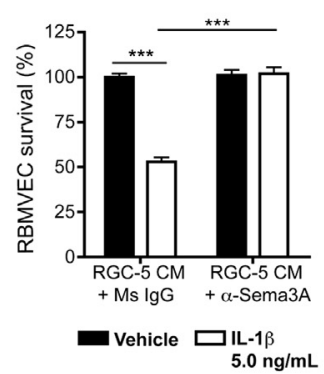

$\mathbf{F}$

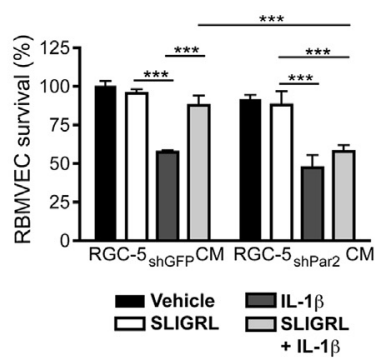

\section{G}
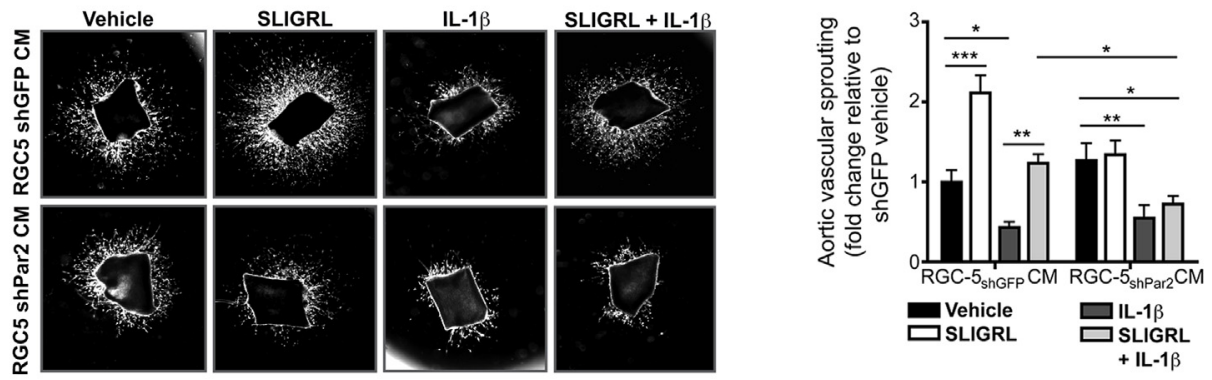

Figure 6 Activation of proteinase-activated receptor (Par)-2 abolishes IL-1 $\beta$-mediated semaphorin 3A (Sema3A) release, reducing endothelial cell death and promoting vascular sprouting. A: Western blot analysis of Sema3A in postnatal day 9 (P9) wild-type (WT) mice whole retinas exposed to oxygen-induced retinopathy (OIR) and injected intravitreally. OIR exposure augments Sema3A expression in P9 vehicle-injected animals; however, 0IR retinas treated with $100 \mu$ mol SLIGRL have significantly reduced Sema3A levels compared with vehicle-injected eyes (2 retinas per group). The bar graph shows densitometry quantification. Conversely, Lv shPar2 treatment augments Sema3A levels compared with Lv shRNA against green fluorescent protein (shGFP)-injected retinas. B: Immunohistochemical analysis on P9 WT mice retina exposed to hyperoxia reveals significant immunoreactivity to Sema3A, particularly in the ganglion cell layer (middle panels) compared to normoxic-exposed retinas (upper panels). Treatment with SLIGRL, however, significantly reduces Sema3A levels in this layer (lower panels). C: Retinal ganglion cell (RGC)-5 cell lines stimulated with IL-1 $\beta$ exhibit time-dependent increases in Sema3a mRNA. D: Control Lv shGFP-infected RGC-5 stimulated with $100 \mu \mathrm{mol} / \mathrm{L}$ SLIGRL abrogates IL-1 $\beta$-dependent increases in Sema3a mRNA after 24 hours; however, this effect is abolished in Lv shPar2-infected RGC-5. E: MTT cell survival assay on rat brain microvascular endothelial cells (RBMVECS) exposed to conditioned media (CM) from RGC- 5 cell lines stimulated with IL-1 $\beta$ reveals significant reduction in endothelial cell survival. Treatment with an antibody to Sema3A abrogates this effect. F: Similar experiment as in E but using CM from Lv shGFP- or Lv shPar2-infected RGC-5 stimulated with IL-1 $\beta$, SLIGRL, or both. IL-1 $\beta$-stimulated CM significantly reduces endothelial cell survival, whereas CM from Lv shGFP RGC-5 co-stimulated with SLIGRL and IL-1 $\beta$ reverses this effect. Lv shPar2 RGC-5 co-stimulated with SLIGRL and IL-1 $\beta$ does not salvage the IL-1 $\beta$-induced endothelial cell death. G: Aortic explants exposed to the same CM as in F. Explants treated with CM from SLIGRL-stimulated RGC-5 have increased sprouting, whereas explants exposed to CM from IL-1 $\beta$-stimulated RGC-5 exhibit diminished sprouting growth. CM from Lv shGFP RGC-5 co-stimulated with SLIGRL and IL-1 $\beta$ translate to normal aortic sprouting angiogenesis, whereas CM from Lv shPar2 RGC-5 does not. The bar graph shows quantification of aortic vascular sprouting. $n=3$ independent experiments $(\mathbf{A}) ; n=6(\mathbf{C}) ; n=7$ to $8(\mathbf{D}) ; n=5$ to $6(\mathbf{E}) ; n=6(\mathbf{F}) ; n=6$ to 9 (G). ${ }^{*} P<0.05, * * P<0.01$, and $* * * P<0.001$. Scale bar $=50 \mu \mathrm{m}(\mathbf{B})$. Original magnification: $\times 300(\mathbf{B}) ; \times 40(\mathbf{G})$. GCL, ganglion cell layer; INL; inner nuclear layer; IPL, inner plexiform layer; $\mathrm{NFL}$, nerve fiber layer; $\mathrm{ONL}$, outer nuclear layer; $\mathrm{OPL}$, outer plexiform layer. 


\section{Oxygen-Induced Retinopathy}

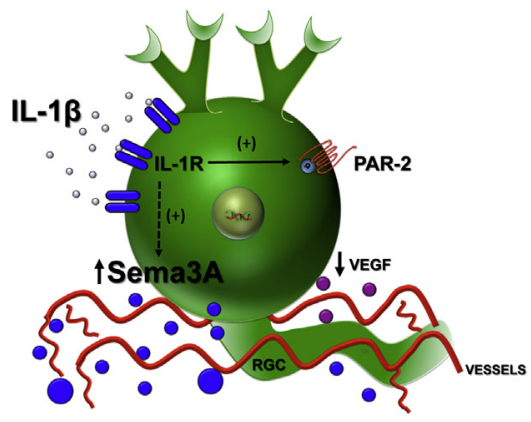

VASO-OBLITERATION

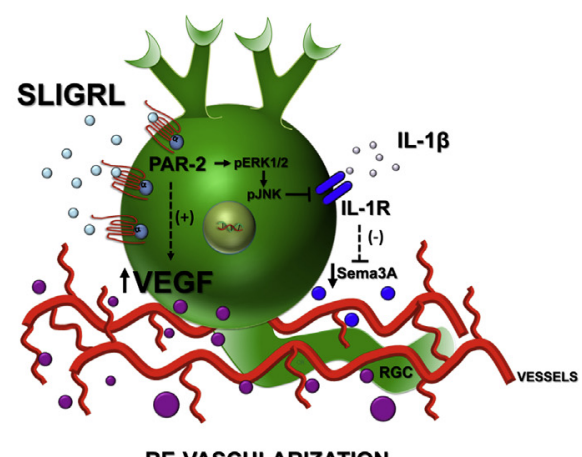

RE-VASCULARIZATION
Figure 7 Proteinase-activated receptor (Par)-2 on retinal ganglion cells (RGCs) enhances revascularization by suppressing IL-1 receptor type I (IL-1RI). Augmented levels of secreted IL-1 $\beta$ stimulate rapid production of Par2 to counteract the hyperoxic stress. However, exhaustive inflammation causes RGCs to suspend Par2 production and instead induces semaphorin $3 \mathrm{~A}$ (Sema3A) production, which leads to vasoobliteration and contributes to pathological preretinal neovascularization. Conversely, timely activation of Par2, using peptide agonist SLIGRL, abrogates this process by suppressing IL-1RI via Erk1/2 and Jnk-dependent processes in RGCs to blunt Sema3A production and concomitantly stimulate vascular endothelial growth factor (VEGF) production to augment the revascularization process of the ischemic retina. normal revascularization of the retina (Figure 2C). We therefore set out to determine how Par2 promotes retinal revascularization in OIR by examining its modulation of IL-1 signaling. We determined IL-1 $\beta$ and IL-1RI levels in retinas from P9 WT mouse pups exposed to OIR and injected intravitreally with SLIGRL or Lv shPar2. Both IL-1 $\beta$ and IL-1RI expression increased in vehicle-injected retinas of animals exposed to hyperoxia (Figure 4, A and B). Par2 stimulation with SLIGRL attenuated the OIR-provoked increase in IL-1RI expression predominantly localized on RGC (Figure 4, A and B); normoxic animals treated with SLIGRL also exhibited moderate reductions in IL-1RI (Supplemental Figure S6, A and B). Conversely Lv shPar2 further augmented IL-1RI expression in OIR (Figure 4A). SLIGRL and Lv shPar2 had no effect on IL-1 $\beta$ expression (Figure $4 \mathrm{~A}$ ).

To determine whether inhibition of IL-1 $\beta$ and stimulation of Par2 acted synergistically in enhancing revascularization, WT mice subjected to OIR were treated with IL-1Ra, SLIGRL, or both. The effects on vaso-obliteration were comparable (Figure 4C), suggesting that Par2 stimulation did not induce any additional mechanism to that through IL-1RI suppression to enhance retinal revascularization in OIR. As anticipated, Par2 $2^{-1-}$ mice were only responsive to IL-1Ra (Supplemental Figure S6C). Consistent with comparable vaso-obliteration in $\mathrm{WT}$ and $\mathrm{Par} 2^{-1-}$ mice (Supplemental Figure S3A), Par2 ${ }^{-1-}$ mice had similar IL1RI levels compared with both normoxic and OIR WT mice, supporting the presence of compensatory mechanism in $\mathrm{Par}^{-1-}$ mice with OIR (Supplemental Figure S6D).

\section{Par2 Stimulation Reduces IL-1RI Expression in Retinal Neurons via Erk1/2 and Downstream Jnk Signaling}

To elucidate signaling pathways by which Par2 regulates IL-1RI expression, we corroborated our in vivo observations on cultured cells. In RGC-5 samples, Par2 stimulation with SLIGRL suppressed time and dose dependently IL-1RI mRNA and protein expression (Figure 5, A and B); concordantly, SLIGRL also attenuated IL-1 $\beta$-induced Irak-1 phosphorylation (Supplemental Figure S7A). Cells infected with Lv shPar2 were unresponsive to SLIGRL (Figure 5, A and B, and Supplemental Figure S7B). Interestingly, in contrast to RGCs, in RBMVECs IL-1RI mRNA and protein expression increased at 1 hour and 2 hours after stimulation with SLIGRL, respectively (Supplemental Figure S7, C and D), suggesting distinct signaling pathways in different cell types.

Par2 stimulation results in activation of several downstream effectors, including $\mathrm{Ca}^{2+}$ transients and mitogenactivated protein kinase (MAPK) signaling. ${ }^{16,42,43}$ RGC-5 samples treated with SLIGRL exhibited a time-dependent increase in MAPK signaling, including Erk1/2, Jnk, and p38 phosphorylation (Figure 5C). Increased MAPK activation was also detected in retina of WT mice subjected to OIR (Supplemental Figure S7E); similar observations were made in Par2 $^{-1-}$ mice, again consistent with compensatory mechanisms in these knockout mice. Inhibition of p38 (using SB203580) in RGC-5 samples did not affect SLIGRLdependent suppression of IL-1RI mRNA, whereas pretreatment with MEK1/2 inhibitor (U0125) or Jnk inhibitor (SP600125) prevented SLIGRL-induced IL-1RI mRNA suppression (Figure 5D). Western blot analysis revealed that the selective MEK1/2 inhibitor (U0125) inhibited Erk1/2 and Jnk phosphorylation, whereas the specific Jnk inhibitor (SP600125) only inhibited Jnk phosphorylation. Together these observations suggest that Par2 stimulation suppresses IL-1RI in RGCs by signaling sequentially via Erk1/2 and Jnk (Figure 5E), as previously reported. ${ }^{44}$ In contrast, in RBMVEC SLIGRL-induced IL-1RI up-regulation was unaffected by the MAPK kinase inhibitor (PD98059) substantiating distinct signaling pathways of Par-2 in different cells types (Supplemental Figure S7, F and G).

\section{Par2 Activation Inhibits IL-1 $\beta$-Mediated Sema3A Release and Ensuing Endothelial Cell Death and Promotes Angiogenesis by Concomitant VEGF Release}

OIR-triggered increase in IL-1 $\beta$ leads to Sema3A release and ensuing endothelial cell death, resulting in increased 
vasoobliteration $^{8,11}$ and hindered normal revascularization. ${ }^{11}$ Because Par2 negatively regulates IL-1RI signaling (Figures 4 and 5), we proceeded to investigate whether this was associated with suppressed Sema3A expression. OIRtriggered Sema3A expression was indeed suppressed by Par2 stimulation with SLIGRL (Figure 6, A and B); as expected, Lv shPar2 further augmented Sema3A levels compared with Lv shGFP (Figure 6A). Concomitantly, VEGF-A levels increased sharply in RGCs on SLIGRL stimulation (Supplemental Figure S8, A and B), facilitating angiogenesis (Supplemental Figure S8, C and D).

To ascertain in vivo findings, we stimulated RGC-5 with IL- $1 \beta$ and measured Sema3A in the presence or absence of SLIGRL. Sema3A mRNA was markedly induced by IL- $1 \beta$ (Figure 6C), and this effect was abrogated by SLIGRL, which was ineffective in Lv shPar2-infected cells (Figure 6D); importantly, SLIGRL in the absence of IL- $1 \beta$ did not affect Sema3A expression.

Next, we determined the functional role of Par2 in angiogenesis by assessing RBMVEC survival, using conditioned media from RGC-5 stimulated with IL- $1 \beta$ in the presence or absence of SLIGRL. Endothelial cell death triggered by IL- $1 \beta$ stimulation of RGC- 5 was Sema3A dependent because this was abrogated using a neutralizing antibody to Sema3A (Figure 6E). IL-1 $\beta$-induced endothelial cell death was also abolished by Par2 stimulation (Figure 6F). Similarly, conditioned media from Par2 stimulated RGC-5 elicited aortic explant vascular sprouting (Figure 6G) due to concomitant VEGF-A release from these cells (Supplemental Figure S8, C and D), whereas conditioned media from IL-1 $\beta$ stimulated RGC-5 diminished aortic explant vascular sprouting, and this effect was abolished by co-treatment of RGC-5 with SLIGRL (Figure 6G). Altogether these data indicate that the actions of Par2 stimulation in the retina negatively modulate IL-1 $\beta$ actions and thus prevent IL- $1 \beta$-induced Sema 3 A release while maintaining elevated levels of VEGF-A; these effects dampen endothelial cell death during oxygen-induced vasoobliteration and augment desirable revascularization through retinovascular proliferation.

\section{Discussion}

Sustained inflammation is an established cause of vessel network injury. ${ }^{5-10,36,45}$ Resident microglial cells, a dominant source of proinflammatory IL- $1 \beta$, are autoactivated by this cytokine, which contributes to exacerbating vaso-obliteration in ischemic retinopathies. ${ }^{8}$ Despite this autoamplified inflammation, vaso-obliteration eventually ceases to progress, giving rise instead to normal retinal revascularization,,${ }^{12,46}$ implying the presence of an intrinsic regulatory mechanism. We found that neuronal Par2 is an important negative regulator of IL-1 $\beta$-induced vascular degeneration, thereby fostering the revascularization of vaso-obliterated retinal tissue. In this context, rapid IL-1 $\beta$ release induces Par2 expression, which in turn negatively regulates IL-1RI signaling by abrogating its expression; these effects essentially counteract the relentless inflammatory autoamplification cascade ${ }^{13,14}$ that would otherwise cause irreversible retinal damage. ${ }^{8,11}$ IL-1RI downregulation occurs in Par2-expressing RGCs where it curtails proapoptotic Sema3A production and concomitantly enhances VEGF secretion, together promoting revascularization of vaso-obliterated retinal regions (Figure 7).

There is increasing evidence that neurons play a crucial role in the formation of retinal vessel network in development and disease. For instance, mice pups that lack retinal ganglion cells (brn3b $b^{Z-d t a /+}$; six 3 -cre mice) are devoid of a retinal capillary network. ${ }^{28}$ Early in OIR, neurons appear to contribute to neurovascular survival by increasing proangiogenic signals, such as GPR91, VEGF, and Netrin1, in an attempt to offset damages from ischemic stress. ${ }^{11,28,32}$ However if unchecked, sustained hypoxia and inflammation can result in suppression of VEGF and secretion of vasorepulsive cue Sema3A, in addition to activation of endoplasmic reticulum stress pathways that translates into an inositol-requiring enzyme-1 $\alpha$-dependent cleavage of Netrin1 mRNA. ${ }^{8,11,32}$ Together, these molecular events divert metabolic stores away from perishing tissue to preserve more viable retinal regions. ${ }^{11}$ Once perishing tissues have been sequestered, mechanisms to prevent the relentless inflammatory destruction of the retina must exist to initiate neurovascular retinal repair. We found that amplified IL-1 $\beta$ signals for increased Par2 expression in RGCs, likely as an attempt to overcome excessive vascular decay and initiate revascularization. In this regard, neuronal Par2 functions as an intrinsic checkpoint to avoid excess proinflammatory cytokine signaling. Specifically, Par2 exerts its beneficial effects by suppressing IL-1RI expression in RGCs via an Erk/Jnkdependent pathway, which in turn limits IL-1 $\beta$-induced Sema3A secretion, despite high IL-1 $\beta$ retinal levels during OIR. Altogether this study highlights a central role for Par2 and uncovers an important regulatory mechanism in neurons that avoids excessive vascular decay and reestablishes normal retinal revascularization in these conditions.

Par2 is ubiquitously expressed in various organs and can have distinct cellular functions depending on the cell type where it is expressed. ${ }^{47,48}$ We found that Par2 in the retina is most prominently localized on RGCs and to a lesser extent on endothelium, where it exhibits functionally distinct cellular processes. Tissue factor, which is expressed on endothelium (but not on neurons), has been found, when constitutively activated, to robustly stimulate angiogenesis of the vascular plexus during development via Par $2^{18}$; however, in OIR, endothelial Par2 activation by tissue factor fails to curb oxygen-induced vaso-obliteration and subsequent pathologic neovascularization. ${ }^{17}$ Correspondingly, we found that activation of Par2 on endothelial cells increases IL-1RI (and IL-1 $\beta^{20}$ ) expression, which may potentiate vascular injury, ${ }^{8}$ and does so seemingly via distinct intracellular signaling pathways. Neuronal Par2, on the other hand, dampens oxygen-induced endothelial cell death by 
curbing IL-1 $\beta$-mediated Sema3A release while maintaining elevated levels of VEGF in the retina, thus allowing normal regrowth of intraretinal neovessels (and consequently avoiding extraretinal neovessel formation). The exact reason why this dichotomy exists in the retina, however, remains elusive. We hypothesize that in pathologic conditions (as seen in ischemic retinopathy), endothelial Par2 does not account for the exaggerated immunologic response and, rather than counteracting this response, exacerbates vascular decay, whereas neuronal Par2 functions as a regulator of inflammation to avoid excessive neurovascular degeneration. In the brain and other organs, a similar dichotomy is apparent; Par2 activation can have opposing effects, depending on the tissue or cell where it is expressed. ${ }^{49,50}$ For instance, experimental models of neurodegenerative disorders reveal that Par2 on glial cells evoke distinct cellular mechanisms from neuronal Par2 to initiate neurodegeneration and neuroprotection, respectively. ${ }^{22,51-54}$

Importantly, this study also highlights the drawback associated with embryonic gene deletion. As previously reported, ${ }^{17}$ OIR-induced vaso-obliteration and neovascularization were unaltered in mice having embryonic deletion of the Par2 gene. We found that conditionally knocking down Par2 in wild-type mice retina invokes a distinct vascular phenotype compared with $\mathrm{Par} 2^{-/-}$mice, whereupon they are exposed to OIR. Furthermore, mice conditionally knocked down for Par2 display divergent molecular signatures with regard to Par2 $2^{-1-}$ mice, indicating that embryonic gene deletion may, in some circumstances, unobtrusively induce compensatory mechanisms that may guise the native function of genes under investigation.

Overall, our findings uncover an important unprecedented functional mechanism induced by inflammation to limit vascular injury. We hereby identify Par2 as an important physiologic mediator that regulates the inflammatory hypoxicischemic retinal environment in ischemic retinopathies, thus allowing for normal vascular regeneration of the retina. To date considerable attention has been focused on inhibiting pathologic neovascularization, ${ }^{55-58}$ whereby anti-VEGF administration is currently accepted as a possible treatment regimen for patients with ischemic retinopathies. ${ }^{59}$ However, there are no clinical treatments that prompt desirable revascularization in an effort to reduce ischemic stress that is crucial to neuroretinal dysfunction and preretinal neovascularization. The current study offers an approach for alleviating ischemic stress using Par2 agonists.

\section{Acknowledgments}

We acknowledge Hensy Fernandez and Isabelle Lahaie for valuable technical assistance. We thank Dr. Christian Beauséjour for providing us with Lv constructs.

N.S., J.C.R., J.-S.J., and S.C. conceived and designed the experiments; N.S., J.C.R., B.N., M.B.-A., K.Z., S.O., D.H., and T.Z. performed the experiments; N.S., J.C.R., and S.C. analyzed the data; J.C.R., P.H., P.S., and J.-S.J. provided expert advice; N.S. and J.C.R prepared the figures; N.S., J.C.R., and S.C. wrote the manuscript.

\section{Supplemental Data}

Supplemental material for this article can be found at http://dx.doi.org/10.1016/j.ajpath.2014.10.020.

\section{References}

1. Yau JWY, Rogers SL, Kawasaki R, Lamoureux EL, Kowalski JW, Bek T, et al; The Meta-Analysis for Eye Disease (Meta-eye) Study Group: Global prevalence and major risk factors of diabetic retinopathy. Diabetes Care 2012, 35:556-564

2. Gilbert C, Rahi J, Eckstein M, O'Sullivan J, Foster A: Retinopathy of prematurity in middle-income countries. Lancet 1997, 350:12-14

3. Sapieha P, Hamel D, Shao Z, Rivera JC, Zaniolo K, Joyal JS, Chemtob S: Proliferative retinopathies: angiogenesis that blinds. Int J Biochem Cell Biol 2010, 42:5-12

4. Fulton A, Hansen R, Moskowitz A, Akula J: The neurovascular retina in retinopathy of prematurity. Prog Retin Eye Res 2009, 28: 452-482

5. Demircan N, Safran BG, Soylu M, Ozcan AA, Sizmaz S: Determination of vitreous interleukin-1 (il-1) and tumour necrosis factor (tnf) levels in proliferative diabetic retinopathy. Eye (Lond) 2006, 20: $1366-1369$

6. Mocan MC, Kadayifcilar S, Eldem B: Elevated intravitreal interleukin-6 levels in patients with proliferative diabetic retinopathy. Can J Ophthalmol 2011, 41:747-752

7. Krady JK, Basu A, Allen CM, Xu Y, LaNoue KF, Gardner TW, Levison SW: Minocycline reduces proinflammatory cytokine expression, microglial activation, and caspase-3 activation in a rodent model of diabetic retinopathy. Diabetes 2005, 54:1559-1565

8. Rivera JC, Sitaras N, Noueihed B, Hamel D, Madaan A, Zhou T, Honore J, Quiniou C, Joyal J, Hardy P, Sennlaub F, Lubell W, Chemtob S: Microglia and IL-1 $\beta$ in ischemic retinopathy elicit microvascular degeneration through neuronal Semaphorin3A. Arterioscler Thromb Vasc Biol 2013, 133:1881-1891

9. dell'Omo R, Semeraro F, Bamonte G, Cifariello F, Romano MR, Costagliola C: Vitreous mediators in retinal hypoxic diseases. Mediators Inflamm 2013:1-16

10. Ishida S, Yamashiro K, Usui T, Kaji Y, Ogura Y, Hia T, Honda Y, Oguchi Y, Adamis A: Leukocytes mediate retinal vascular remodeling during dvelopment and vaso-obliteration in disease. Nat Med 2003, 9:781-789

11. Joyal JS, Sitaras N, Binet F, Rivera JC, Stahl A, Zaniolo K, Shao Z, Polosa A, Zhu T, Hamel D, Djavari M, Kunik D, Honoré JC, Picard E, Zabeida A, Varma DR, Hickson G, Mancini J, Klagsbrun M, Costantino S, Beauséjour C, Lachapelle P, Smith LE, Chemtob S, Sapieha P: Ischemic neurons prevent vascular regeneration of neural tissue by secreting semaphorin 3A. Blood 2011, 117: 6024-6035

12. Dorfman A, Dembinska O, Chemtob S, Lachapelle P: Early manifestations of postnatal hyperoxia on the retinal structure and function of the neonatal rat. Invest Ophthalmol Vis Sci 2008, 49:458-466

13. Teshima S, Nakanishi H, Nishizawa M, Kitagawa K, Kaibori M, Yamada M, Habara K, Kwon A, Kamiyama Y, Ito S, Okumura T: Up-regulation of IL-1 receptor through PI3K/Akt is essential for the induction of iNOS gene expression in hepatocytes. J Hepatol 2004 40:616-623

14. Skundric DS, Bealmear B, Lisak RP: Induced upregulation of IL-1, IL-1RA and IL-1R type I gene expression by Schwann cells. J Neuroimmunol 1997, 74:9-18 
15. Kowluru R, Zhong Q, Santos J: Matrix metalloproteinases in diabetic retinopathy: potential role of MMP-9. Expert Opin Investig Drugs 2012, 21:797-805

16. Soh UJK, Dores MR, Chen B, Trejo J: Signal transduction by protease-activated receptors. Br J Pharmacol 2010, 160:191-203

17. Uusitalo-Jarvinen H, Kurokawa T, Mueller BM, Andrade-Gordon P, Friedlander M, Ruf W: Role of protease activated receptor 1 and 2 signaling in hypoxia-induced angiogenesis. Arterioscler Thromb Vasc Biol 2007, 27:1456-1462

18. Belting M, Dorrell MI, Sandgren S, Aguilar E, Ahamed J, Dorfleutner A, Carmeliet P, Mueller BM, Friedlander M, Ruf W: Regulation of angiogenesis by tissue factor cytoplasmic domain signaling. Nat Med 2004, 10:502-509

19. Tsai SH, Sheu MT, Liang YC, Cheng HT, Fang SS, Chen C: TGF- $\beta$ inhibits IL-1 $\beta$-activated PAR-2 expression through multiple pathways in human primary synovial cells. J Biomed Sci 2009, 16 : 97-111

20. Ritchie E, Saka M, MacKenzie C, Drummond R, Wheeler-Jones C, Kanke T, Plevin R: Cytokine upregulation of proteinase-activatedreceptors 2 and 4 expression mediated by p38 MAP kinase and inhibitory kappa B kinase $\beta$ in human endothelial cells. Br J Pharmacol 2007, 150:1044-1054

21. Boileau C, Amiable N, Martel-Pelletier J, Fahmi H, Duval N, Pelletier J: Activation of proteinase-activated receptor 2 in human osteoarthritic cartilage upregulates catabolic and proinflammatory pathways capable of inducing cartilage degradation: a basic science study. Arthritis Res Ther 2007, 9:R121

22. Noorbakhsh F, Vergnolle N, McArthur JC, Silva C, AndradeGordon P, Vodjgani M, Hollenberg M, Power C: Protease-activated receptor-2 induction by neuroinflammation prevents neuronal death during HIV infection. J Immunol 2005, 174:7320-7329

23. Suharti C, van Gorp EC, Setiati TE, Dolmans WM, Djokomoeljanto RJ, Hack CE, Hugo ten C, van der Meer JW: The role of cytokines in activation of coagulation and fibrinolysis in dengue shock syndrome. Thromb Haemost 2002, 87:42-46

24. Smith LE, Wesoloiuski E, McLellan A, Kostyk SK, D'Amato R, Sullivan R, D'Amore PA: Oxygen-induced retinopathy in the mouse. Invest Ophthalmol Vis Sci 1994, 35:101-111

25. Stahl A, Connor KM, Sapieha P, Chen J, Dennison RJ, Krah NM, Seaward MR, Willett KL, Aderman CM, Guerin KI, Hua J, Lofqvist C, Hellstrom A, Smith LE: The mouse retina as an angiogenesis model. Invest Ophthalmol Vis Sci 2010, 51: 2813-2826

26. Stahl A, Connor KM, Sapieha P, Willett KL, Krah NM, Dennison RJ, Chen J, Guerin KI, Smith LE: Computer-aided quantification of retinal neovascularization. Angiogenesis 2009, 12:297-301

27. Dull T, Zufferey R, Kelly M, Mandel RJ, Nguyen M, Trono D, Naldini L: A third-generation lentivirus vector with a conditional packaging system. J Virol 1998, 72:8463

28. Sapieha P, Sirinyan M, Hamel D, Zaniolo K, Joyal JS, Cho JH, Honoré JC, Kermorvant-Duchemin E, Varma DR, Tremblay S, Leduc M, Rihakova L, Hardy P, Klein WH, Mu X, Mamer O, Lachapelle P, Di Polo A, Beauséjour C, Andelfinger G, Mitchell G, Sennlaub F, Chemtob S: The succinate receptor GPR91 in neurons has a major role in retinal angiogenesis. Nat Med 2008, 14: 1067-1076

29. Chang-Ling T, Neill AL, Hunt NH: Early microvascular changes in murine cerebral malaria detected in retinal wholemounts. Am J Pathol 1992, 140:1121-1130

30. Barbaric I, Miller G, Dear T: Appearances can be deceiving: phenotypes of knockout mice. Brief Funct Genomic Proteomic 2007, 6: 91-103

31. Schmidlin F, Amadesi S, Dabbagh K, Lewis DE, Knott P, Bunnet NW, Gater PR, Geppetti P, Bertrand C, Stevens ME: Protease-activated receptor 2 mediates eosinophil infiltration and hyperreactivity in allergic inflammation of the airway. J Immunol 2002, 169:5315-5321
32. Binet F, Mawambo G, Sitaras N, Tetreault N, Lapalme E, Favret S, Cerani A, Leboeuf D, Tremblay S, Rezende F, Juan A, Stahl A, Joyal J, Milot É, Kaufman R, Guimond M, Kennedy T, Sapieha P: Neuronal ER stress impedes myeloid-cell-induced vascular regeneration through ire1-alpha degradation of netrin-1. Cell Metab 2013, $17: 353-371$

33. Cerani A, Tetreault N, Menard C, Lapalme E, Patel C, Sitaras N, Beaudoin F, Leboeuf D, De Guire V, Binet F, Dejda A, Rezende F, Miloudi K, Sapieha P: Neuron-derived Semaphorin 3A is an early inducer of vascular permeability in diabetic retinopathy via neuropilin-1. Cell Metab 2013, 18:505-518

34. Kaufmann R, Hollenberg M: Proteinase-activated receptors (PARs) and calcium signaling in cancer. Adv Exp Med Biol 2012, 740:979-1000

35. Kim Y, West XZ, Byzova TV: Inflammation and oxidative stress in angiogenesis and vascular disease. J Mol Med 2013, 91:323-328

36. Gardiner TA, Gibson DS, de Gooyer TE, de la Cruz VF, McDonald DM, Stitt A: Inhibition of tumor necrosis factor- $\alpha$ improves physiological angiogenesis and reduces pathological neovascularization in ischemic retinopathy. Am J Pathol 2005, 166: 637-644

37. Meyer N, Christoph J, Makrinioti H, Indermitte P, Rhyner C, Soyka M, Eiwegger T, Chalubinski T, Wanke K, Fujita H, Wawrzyniak P, Burgler S, Zhang S, Akdis M, Menz G, Akdis C: Inhibition of angiogenesis by IL-32: possible role in asthma. J Allergy Clin Immunol 2012, 129:964-973.e7

38. Massague J, Blain S, Lo R: TGF-beta signaling in growth control, cancer, and heritable disorders. Cell 2000, 103:295-309

39. Kermorvant-Duchemin E, Sennlaub F, Sirinyan M, Brault S, Andelfinger G, Kooli A, Germain S, Ong H, d'Orleans-Juste P, Gobeil F, Zhu T, Boisvert C, Hardy P, Jain K, Falck JR, Balazy M, Chemtob S: Trans-arachidonic acids generated during nitrative stress induce a thrombospondin-1-dependent microvascular degeneration. Nat Med 2005, 11:1339-1345

40. Minuzzo S, Moserle L, Indraccolo S, Amadori A: Angiogenesis meets immunology: cytokine gene therapy of cancer. Mol Aspects Med 2007, 28

41. Shen H, Yao P, Lee E, Greenhalgh D, Soulika A: Interferon-gamma inhibits healing post scald burn injury. Wound Repair Regen 2012 , 20:580-591

42. Rothmeier A, Ruf W: Protease-activated receptor 2 signaling in inflammation. Semin Immunopathol 2012, 34:133-149

43. Ossovskaya VS, Nunnet N: Protease-activated receptors: contribution to physiology and disease. Physiol Rev 2004, 84:579-621

44. Paumelle R, Tulasne D, Leroy C, Coll J, Vandenbunder B, Fafeur V: Sequential activation of ERK and repression of JNK by scatter factor/hepatocyte growth factor in Madin-Darby canine kidney epithelial cells. Mol Biol Cell 2000, 11:3751-3763

45. Tremblay S, Miloudi K, Chaychi S, Favret S, Binet F, Polosa A, Lachapelle P, Chemtob S, Sapieha P: Systemic inflammation perturbs developmental retinal angiogenesis and neuroretinal function. Invest Ophthalmol Vis Sci 2013, 54:8125-8139

46. Lange C, Ehlken C, Stahl A, Martin G, Hansen L, Agostini H: Kinetics of retinal vaso-obliteration and neovascularisation in the oxygen-induced retinopathy (OIR) mouse model. Graefes Arch Clin Exp Ophthalmol 2009, 247:1205-1211

47. Bucci M, Roviezzo F, Cirino G: Protease-activated receptor-2 (PAR2) in cardiovascular system. Vascul Pharmacol 2005, 43: 247-253

48. Ramachandran R, Hollenberg MD: Proteinases and signalling: pathophysiological and therapeutic implications via PARs and more. Br J Pharmacol 2008, 153:S263-S282

49. Luo W, Wang Y, Reiser G: Protease-activated receptors in the brain: receptor expression, activation, and functions in neurodegeneration and neuroprotection. Brain Res Rev 2007, 56:331-345

50. Ramachandran R, Noorbakhsh F, DeFea K, Hollenberg MD: Targeting proteinase-activated receptors: therapeutic potential and challenges. Nat Rev Drug Discov 2012, 11:69-86 
51. Afkhami-Goli A, Noorbakhsh F, Keller AJ, Vergnolle N, Westaway D, Jhamandas JH, Andrade-Gordon P, Hollenberg MD, Arab H, Dyck RH, Power C: Proteinase-activated receptor-2 exerts protective and pathogenic cell type-specific effects in Alzheimer's disease. J Immunol 2007, 179:5493-5503

52. Noorbakhsh F, Tsutsui S, Vergnolle N, Boven LA, Shariat N, Vodjgani M, Warren KG, Andrade-Gordon P, Hollenberg MD, Power C: Proteinase-activated receptor 2 modulates neuroinflammation in experimental autoimmune encephalomyelitis and multiple sclerosis. J Exp Med 2006, 203:425-435

53. Jin G, Hayashi T, Kawagoe J, Takizawa T, Nagata T, Nagano I, SyojI M, Abe K: Deficiency of PAR-2 gene increases acute focal ischemic brain injury. J Cereb Blood Flow Metab 2005, 25: 302-313
54. Wang H, Ubl J, Reiser G: Four subtypes of protease-activated receptors, co-expressed in rat astrocytes, evoke different physiological signaling. Glia 2002, 37:53-63

55. Hellström A, Smith LE, Dammann O: Retinopathy of prematurity. Lancet 2013, 382:1445-1457

56. Miller J, Couter JL, Strauss E, Ferrara N: Vascular endothelial growth factor A in intraocular vascular disease. Ophthalmology 2012, 120:106-114

57. Wang S, Park J, Duh E: Novel targets against retinal angiogenesis in diabetic retinopathy. Curr Diab Rep 2012, 12:355-363

58. Hernandez C, Simo R: Strategies for blocking angiogenesis in diabetic retinopathy: from basic science to clinical practice. Expert Opin Investig Drugs 2007, 16:1209-1226

59. Stewart MW: The expanding role of vascular endothelial growth factor inhibitors in ophthalmology. Mayo Clin Proc 2012, 87:77-88 